\title{
From NURBS to NURPS geometries
}

\author{
Hendrik Speleers Carla Manni \\ Francesca Pelosi
}

Report TW619, November 2012

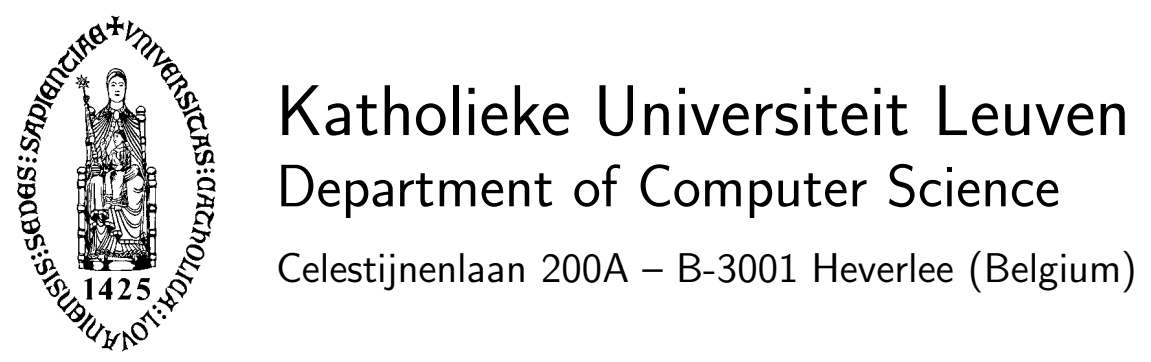




\title{
From NURBS to NURPS geometries
}

\author{
Hendrik Speleers Carla Manni \\ Francesca Pelosi
}

Report TW619, November 2012

Department of Computer Science, K.U.Leuven

\begin{abstract}
Quadratic Powell-Sabin splines and their rational extension, the socalled NURPS surfaces, are an interesting alternative for classical tensor-product NURBS in the context of isogeometric analysis, because they allow the use of local refinements while retaining a Bspline like representation and exact description of conic sections. In this paper we present a simple and effective strategy to convert a given planar geometry defined by a quadratic NURBS representation into a NURPS representation, suitable for the analysis.
\end{abstract}

Keywords : Powell-Sabin splines, NURBS, NURPS, isogeometric analysis. 


\title{
From NURBS to NURPS geometries
}

\author{
Hendrik Speleers ${ }^{\mathrm{a}}$, Carla Manni ${ }^{\mathrm{b}}$, Francesca Pelosi $^{\mathrm{b}}$ \\ ${ }^{a}$ Departement Computerwetenschappen, Katholieke Universiteit Leuven - Belgium \\ ${ }^{b}$ Dipartimento di Matematica, Università di Roma "Tor Vergata" - Italy
}

\begin{abstract}
Quadratic Powell-Sabin splines and their rational extension, the so-called NURPS surfaces, are an interesting alternative for classical tensor-product NURBS in the context of isogeometric analysis, because they allow the use of local refinements while retaining a B-spline like representation and exact description of conic sections. In this paper we present a simple and effective strategy to convert a given planar geometry defined by a quadratic NURBS representation into a NURPS representation, suitable for the analysis.
\end{abstract}

Keywords: Powell-Sabin splines, NURBS, NURPS, isogeometric analysis.

\section{Introduction}

Tensor-product NURBS (Non-Uniform Rational B-Splines) are the undisputed standard in commercial Computer Aided Design (CAD) systems thanks to their description in terms of basis functions possessing the main nice properties of B-splines and to their ability to exactly reproduce conic sections. This fact motivated the interest they are receiving in the context of Isogeometric Analysis (IgA), a new paradigm for the analysis of problems governed by partial differential equations [9], which aims to improve the connection between numerical simulation and CAD systems.

A key ingredient in IgA is to retain along the entire analysis process the exact geometry of the physical domain, which can be ordinarily described by tensor-product NURBS. Unfortunately, the tensor-product approach is not so suitable from the analysis point of view because it precludes strictly localized refinements. For this reason, other spline structures that allow local

Email addresses: hendrik.speleers@cs.kuleuven.be (Hendrik Speleers), manni@mat . uniroma2. it (Carla Manni), pelosi@mat.uniroma2.it (Francesca Pelosi) 
refinements are also investigated in IgA, such as T-splines [1, 4], hierarchical splines [27] and LR-splines [7].

Recently, quadratic Powell-Sabin (PS) splines and their rational counterpart have been proposed as an alternative for the numerical solution of partial differential equations: diffusion problems were addressed in [23], and more challenging advection-diffusion-reaction problems in [25]. PS splines are $C^{1}$ piecewise quadratic polynomials defined on a particular refinement - the so-called PS refinement - of any given triangulation [17]. They are a popular tool in CAGD (Computer Aided Geometric Design) and approximation theory, see, e.g., [6, 14, 20, 28]. PS splines can be represented in terms of basis functions possessing all the nice properties of the classical univariate B-splines, the so-called (normalized) PS B-splines [5]. A rational extension of PS splines, referred to as NURPS (Non-Uniform Rational PS) surfaces, can also be easily defined [24, 29, 30]. NURPS surfaces allow an exact representation of quadrics, and their shape can be locally controlled by control points and weights in a geometrically intuitive way.

Thanks to their structure based on triangulations, PS splines and their rational extension offer the flexibility of classical Finite Element Methods (FEM) with respect to local refinements. Moreover, they share with standard tensorproduct NURBS the increased smoothness, the B-spline like basis, and the ability to exactly represent profiles of interest in engineering applications as conic sections. Therefore, they constitute a natural bridge between classical FEM and NURBS-based IgA.

In practical applications, quadratic NURBS are of particular interest because they provide an exact description of conic sections by using the minimum degree. To completely frame the PS/NURPS approach in the context of $\operatorname{IgA}$, in this paper we focus on the problem of converting the quadratic NURBS representation of a planar domain into a NURPS form. More precisely, we present a simple and effective conversion procedure, which preserves the NURBS parametrization on the boundary. In general, although the geometry of the domain is exactly described, the given parametrization is not maintained in the interior. Nevertheless, it turns out that the provided NURPS parametrization can be made arbitrarily close to the original NURBS one.

Our procedure can also be applied straightforwardly in case the object of interest is a two-dimensional manifold embedded in a multi-dimensional space. Of course, a general quadratic NURBS surface cannot be reproduced by a NURPS surface, but the considered approach provides a very efficient 
and accurate NURPS approximation of the given NURBS geometry, still preserving the parametrization along the boundary. On this concern, we remark that sometimes the given NURBS geometry is already an approximation of the original domain of interest, as illustrated in [2]. Therefore, the obtained NURPS approximation of the true geometry is not necessarily worse than the NURBS one.

The remainder of the paper consists of six sections. In the next one we summarize basic properties of NURBS. The construction and some properties of PS B-splines and NURPS surfaces are addressed in Section 3. Section 4 describes the conversion of a quadratic NURBS representation into an appropriate NURPS form, and Section 5 analyzes how such a conversion behaves along a refinement process. Some numerical experiments are presented in Section 6. We end in Section 7 with some concluding remarks.

\section{B-splines and NURBS}

Although B-splines and NURBS are well known, we recall in this section their definition and salient properties both to fix the notation and to make the presentation self-contained.

Let $0 \leq p \in \mathbb{N}$ be given. Let us consider a sequence of knots

$$
\mathbf{u}:=\left\{u_{1} \leq u_{2} \leq \cdots \leq u_{n+p+1}\right\}, \quad n \in \mathbb{N},
$$

and let us denote by $1 \leq r_{i} \leq p$ the multiplicity of the knot $u_{i}$, i.e.,

$$
\cdots<u_{i}=u_{i+1}=\cdots=u_{i+r_{i}-1}<u_{i+r_{i}} \leq \cdots .
$$

Setting

$$
N_{i, 0, \mathbf{u}}(t):= \begin{cases}1, & \text { if } t \in\left[u_{i}, u_{i+1}\right) \\ 0, & \text { elsewhere }\end{cases}
$$

according to [3], we define the $i$-th (algebraic) B-spline of degree $p$ corresponding to the knot sequence $\mathbf{u}$, as

$$
N_{i, p, \mathbf{u}}(t):=\frac{t-u_{i}}{u_{i+p}-u_{i}} N_{i, p-1, \mathbf{u}}(t)+\frac{u_{i+p+1}-t}{u_{i+p+1}-u_{i+1}} N_{i+1, p-1, \mathbf{u}}(t), \quad p>0 .
$$

Fractions with zero denominators are considered to be zero.

It is well known that B-splines are piecewise polynomial functions, that is $N_{i, p, \mathbf{u}}(t) \in \mathbb{P}_{p}$ for each interval $t \in\left[u_{r}, u_{r+1}\right)$, where $\mathbb{P}_{p}$ denotes the space of polynomials of degree $p$. B-splines possess the following fundamental properties [3]: 
- nonnegativity: $N_{i, p, \mathbf{u}}(t) \geq 0$;

- partition of unity: $\sum_{i=1}^{n} N_{i, p, \mathbf{u}}(t)=1, \quad t \in\left[u_{p+1}, u_{n+1}\right)$;

- compact support: $N_{i, p, \mathbf{u}}(t)=0, \quad t \notin\left[u_{i}, u_{i+p+1}\right]$;

- smoothness: $N_{i, p, \mathbf{u}}(t)$ is $p-r_{j}$ times continuously differentiable at $u_{j}$, being $r_{j}$ the multiplicity of $u_{j}$ in the knot sequence;

- local linear independence: $N_{i-p, p, \mathbf{u}}(t), \ldots, N_{i-1, p, \mathbf{u}}(t), N_{i, p, \mathbf{u}}(t)$ are linearly independent on $\left[u_{i}, u_{i+1}\right)$.

Denoting by

$$
\mathbb{S}_{p, \mathbf{u}}:=\left\{\sum_{j=1}^{n} c_{j} N_{j, p, \mathbf{u}}, c_{j} \in \mathbb{R}\right\},
$$

the linear space spanned by the given B-splines, it is well known that in $\left[u_{p+1}, u_{n+1}\right)$ such space coincides with the space of piecewise polynomials of degree $p$ which are $p-r_{j}$ times continuously differentiable at any $u_{j} \in$ $\left(u_{p+1}, u_{n+1}\right)$. Moreover, the set

$$
\left\{N_{j, p, \mathbf{u}}, j=1, \ldots, n\right\}
$$

provides actually the "best" basis of this space, both from a geometrical and a computational point of view [15]. Roughly speaking, this means that the basis (2) has an optimal condition number and, considering any curve

$$
\mathbf{C}(t):=\sum_{j=1}^{n} \mathbf{c}_{j} N_{j, p, \mathbf{u}}(t), \quad \mathbf{c}_{j} \in \mathbb{R}^{d}
$$

the polygonal line connecting the control points $\mathbf{c}_{j}, j=1, \ldots, n$, mimics the shape of the curve in an optimal way. This property is the reason of the success of B-splines in free form design.

Unfortunately, B-splines are not able to exactly describe conic sections which are shapes of salient interest in several engineering applications. This motivated the introduction of NURBS. The NURBS model is based on rational functions obtained from B-splines as follows

$$
R_{i, p, \mathbf{u}, \mathbf{W}}(t):=\frac{w_{i} N_{i, p, \mathbf{u}}(t)}{\sum_{j=1}^{n} w_{j} N_{j, p, \mathbf{u}}(t)}, \quad i=1, \ldots, n,
$$


where $\mathbf{W}:=\left\{w_{i} \in \mathbb{R}, i=1, \ldots, n\right\}$ are the weights ${ }^{1}$. NURBS inherit all the classical important properties of B-splines, moreover they allow an exact representation of conic sections [16]. A NURBS curve in $\mathbb{R}^{d}$ is therefore a parametric curve of the form

$$
\mathbf{C}^{\mathbf{W}}(t):=\sum_{j=1}^{n} \mathbf{p}_{j} R_{j, p, \mathbf{u}, \mathbf{W}}(t), \quad \mathbf{p}_{j} \in \mathbb{R}^{d}
$$

where the coefficients $\mathbf{p}_{j}$ are called again control points of the curve. Denoting by $p_{j}^{r}, r=1, \ldots, d$, the components of the control point $\mathbf{p}_{j}$, it turns out that $\mathbf{C}^{\mathbf{W}}$ is a projection onto $\mathbb{R}^{d}$ of the B-spline curve

$$
\left(\sum_{j=1}^{n} w_{j} p_{j}^{1} N_{j, p, \mathbf{u}}, \ldots, \sum_{j=1}^{n} w_{j} p_{j}^{d} N_{j, p, \mathbf{u}}, \sum_{j=1}^{n} w_{j} N_{j, p, \mathbf{u}}\right) .
$$

Tensor-product NURBS are the undisputed standard in commercial CAD systems. This fact motivated their use in the context of IgA, see e.g. [9], a new paradigm for the analysis of problems governed by partial differential equations. A tensor-product NURBS surface has the form

$$
\sum_{i=1}^{n} \sum_{j=1}^{m} \mathbf{p}_{i, j} R_{i, j, p, q}(s, t)
$$

where

$R_{i, j, p, q}(s, t):=\frac{w_{i, j} N_{i, p, \mathbf{u}}(s) N_{j, q, \overline{\mathbf{u}}}(t)}{\sum_{k=1}^{n} \sum_{l=1}^{m} w_{k, l} N_{k, p, \mathbf{u}}(s) N_{l, q, \overline{\mathbf{u}}}(t)}, \quad i=1, \ldots, n, \quad j=1, \ldots, m$,

$w_{i, j}$ are positive weights, and $\mathbf{u}, \overline{\mathbf{u}}$ are suitable sequences of knots.

A key ingredient in IgA is to retain along all the analysis process the exact geometry of the physical domain, $\Omega$, which can be usually described by a tensor-product NURBS map. Focusing on bivariate problems we have, see Fig. 1,

$$
\Omega=\mathbf{G}\left(\Omega_{0}\right)
$$

\footnotetext{
${ }^{1}$ Although some negative weights could be used as well, usually only positive values are considered in order to deal with nonnegative basis functions.
} 


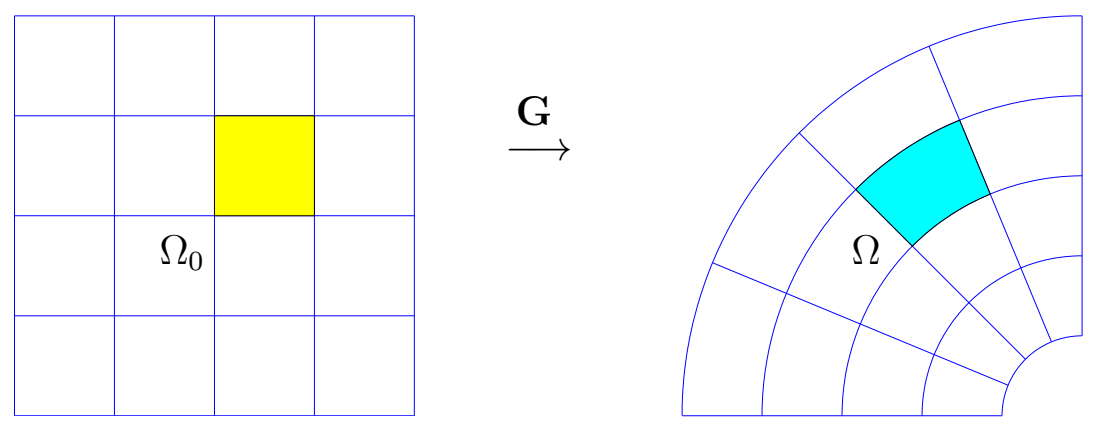

Figure 1: Geometry map.

where $\Omega_{0}=[0,1] \times[0,1]$ is the so-called parametric domain, and $\mathbf{G}$ is the geometry map usually expressed by means of tensor-product NURBS,

$$
\mathbf{G}(s, t):=\sum_{i=1}^{n} \sum_{j=1}^{m} \mathbf{p}_{i, j} R_{i, j, p, q}(s, t), \quad s, t \in[0,1] .
$$

In practical applications, quadratic NURBS are of particular interest because they provide an exact description of conic sections with minimum degree. The aim of this paper is to convert a quadratic NURBS representation of a planar domain into a NURPS form, the rational generalization of PS $\mathrm{B}$-splines. This is mainly motivated by the use of PS splines, and their rational extension, in IgA. These splines combine the flexibility of FEM regarding local refinements, with the high order of smoothness and exact description of conic sections typical for NURBS [25].

Therefore, in the following we only consider quadratic NURBS and, to simplify the notation, we omit the subscript 2 for the degree, i.e., we simply denote

$$
N_{j, \mathbf{u}}(s):=N_{j, 2, \mathbf{u}}(s), \quad R_{i, j}(s, t):=R_{i, j, 2,2}(s, t) .
$$

\section{Quadratic PS B-splines and NURPS surfaces}

In this section we briefly summarize from $[5,24,29]$ the construction and some properties of quadratic B-splines over a Powell-Sabin refinement of a triangulation of a planar domain and their rational generalization.

Let $\Omega_{0}$ be a polygonal domain in $\mathbb{R}^{2}$ and let $\mathcal{T}$ be a triangulation of $\Omega_{0}$. We denote by

$$
\mathbf{V}_{l}:=\left(s_{l}, t_{l}\right), \quad l=1, \ldots, N_{V},
$$

the vertices of the given triangulation. A Powell-Sabin refinement $\mathcal{T}^{*}$ of $\mathcal{T}$ is the refined triangulation obtained by subdividing each triangle of $\mathcal{T}$ into six subtriangles as follows (see also Fig. 2). 

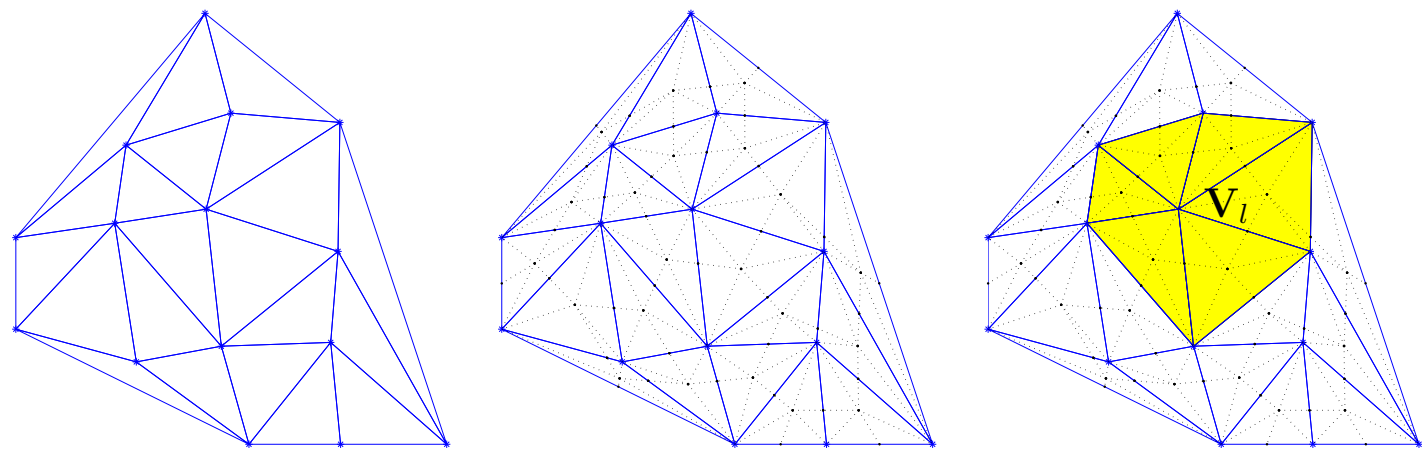

Figure 2: A triangulation $\mathcal{T}$, a Powell-Sabin refinement $\mathcal{T}^{*}$ of $\mathcal{T}$ (in dotted lines), and the molecule $\Omega_{0, l}$ of vertex $\mathbf{V}_{l}$ (shaded, right).

- Select a point, say $\mathbf{C}_{i}$, inside any triangle $\tau_{i}$ of $\mathcal{T}$ so that, if two triangles $\tau_{i}$ and $\tau_{j}$ have a common edge, then the line joining $\mathbf{C}_{i}$ and $\mathbf{C}_{j}$ intersects the common edge at a point $\mathbf{Z}_{i, j}{ }^{2}$

- Join each point $\mathbf{C}_{i}$ to the vertices of $\tau_{i}$.

- For each edge of the triangle $\tau_{i}$

- which belongs to the boundary $\partial \Omega_{0}$ : join $\mathbf{C}_{i}$ to an arbitrary point $\mathbf{Z}_{i, j}^{b}$ on that edge ${ }^{3}$;

- which is common to a triangle $\tau_{j}$ : join $\mathbf{C}_{i}$ to $\mathbf{Z}_{i, j}$.

We denote by

$$
\mathcal{S}_{2}^{1}\left(\mathcal{T}^{*}\right):=\left\{f \in C^{1}\left(\Omega_{0}\right): f_{\mid \tau_{j}^{*}} \in \mathbb{P}_{2}, \tau_{j}^{*} \in \mathcal{T}^{*}\right\}
$$

the space of Powell-Sabin splines [17], i.e. the linear space of $C^{1}$ piecewise quadratic polynomials on $\mathcal{T}^{*}$. It is well known $[5,17,20]$ that:

- $\operatorname{dim}\left(\mathcal{S}_{2}^{1}\left(\mathcal{T}^{*}\right)\right)=3 N_{V}$

- any element of $\mathcal{S}_{2}^{1}\left(\mathcal{T}^{*}\right)$ is uniquely specified by its value and its gradient at the vertices of $\mathcal{T}$, and can be locally constructed on each triangle of $\mathcal{T}$ once these values and gradients are given.

\footnotetext{
${ }^{2}$ Such a choice is always possible: $\mathbf{C}_{i}$ can be selected as the center of the inscribed circle. Usually the barycenter of $\tau_{i}$ is also a valid choice (but not always).

${ }^{3}$ For boundary edges the subscript $j$ refers to the edge.
} 
In [5] B-spline like basis functions $\left\{B_{l}^{(j)}, j=1,2,3, l=1, \ldots, N_{V}\right\}$ of the space $\mathcal{S}_{2}^{1}\left(\mathcal{T}^{*}\right)$ have been determined such that $f=\sum_{l=1}^{N_{V}} \sum_{j=1}^{3} c_{l, j} B_{l}^{(j)}$ for all $f \in \mathcal{S}_{2}^{1}\left(\mathcal{T}^{*}\right)$, and

$$
B_{l}^{(j)}(s, t) \geq 0, \quad \sum_{l=1}^{N_{V}} \sum_{j=1}^{3} B_{l}^{(j)}(s, t)=1, \quad(s, t) \in \Omega_{0} .
$$

The functions $B_{l}^{(j)}$ will be referred to as Powell-Sabin B-splines. To locally construct these PS B-splines $B_{l}^{(j)}, j=1,2,3$, with support in the molecule $\Omega_{0, l}{ }^{4}$ of vertex $\mathbf{V}_{l}$, it suffices to specify their values and gradients at any vertex of $\mathcal{T}$. Due to the structure of the support $\Omega_{0, l}$ we have

$$
B_{l}^{(j)}\left(\mathbf{V}_{k}\right)=0, \quad \frac{\partial}{\partial s} B_{l}^{(j)}\left(\mathbf{V}_{k}\right)=0, \quad \frac{\partial}{\partial t} B_{l}^{(j)}\left(\mathbf{V}_{k}\right)=0
$$

for any vertex $\mathbf{V}_{k} \neq \mathbf{V}_{l}$. We denote

$$
B_{l}^{(j)}\left(\mathbf{V}_{l}\right)=: \alpha_{l}^{(j)}, \quad \frac{\partial}{\partial s} B_{l}^{(j)}\left(\mathbf{V}_{l}\right)=: \beta_{l}^{(j)}, \quad \frac{\partial}{\partial t} B_{l}^{(j)}\left(\mathbf{V}_{l}\right)=: \gamma_{l}^{(j)} .
$$

For each vertex $\mathbf{V}_{l}$, let us determine three points

$$
\left\{\mathbf{Q}_{l}^{(j)}=\left(Q_{l, s}^{(j)}, Q_{l, t}^{(j)}\right), j=1,2,3\right\}
$$

such that, for $l=1, \ldots, N_{V}$,

$$
\left(\begin{array}{ccc}
\alpha_{l}^{(1)} & \alpha_{l}^{(2)} & \alpha_{l}^{(3)} \\
\beta_{l}^{(1)} & \beta_{l}^{(2)} & \beta_{l}^{(3)} \\
\gamma_{l}^{(1)} & \gamma_{l}^{(2)} & \gamma_{l}^{(3)}
\end{array}\right)\left(\begin{array}{lll}
Q_{l, s}^{(1)} & Q_{l, t}^{(1)} & 1 \\
Q_{l, s}^{(2)} & Q_{l, t}^{(2)} & 1 \\
Q_{l, s}^{(3)} & Q_{l, t}^{(3)} & 1
\end{array}\right)=\left(\begin{array}{ccc}
s_{l} & t_{l} & 1 \\
1 & 0 & 0 \\
0 & 1 & 0
\end{array}\right) .
$$

Therefore, we have

$$
s=\sum_{l=1}^{N_{V}} \sum_{j=1}^{3} Q_{l, s}^{(j)} B_{l}^{(j)}(s, t), \quad t=\sum_{l=1}^{N_{V}} \sum_{j=1}^{3} Q_{l, t}^{(j)} B_{l}^{(j)}(s, t) .
$$

The triangle with vertices $\left\{\mathbf{Q}_{l}^{(j)}, j=1,2,3\right\}$ is called PS triangle associated to the vertex $\mathbf{V}_{l}$ and will be denoted by $T_{l}$.

From (10) it follows that for each vertex $\mathbf{V}_{l}$ the three functions $B_{l}^{(j)}, j=$ $1,2,3$, are uniquely determined by the points $\left\{\mathbf{Q}_{l}^{(j)}, j=1,2,3\right\}$. Moreover,

\footnotetext{
${ }^{4}$ the subset of $\Omega_{0}$ consisting of the points belonging to the union of all triangles of $\mathcal{T}$ containing the vertex $\mathbf{V}_{l}$, see Fig. 2 (right)
} 
- $\left(\alpha_{l}^{(1)}, \alpha_{l}^{(2)}, \alpha_{l}^{(3)}\right)$ are the barycentric coordinates of $\mathbf{V}_{l}$ with respect to the triangle $T_{l}$;

- $\left(\beta_{l}^{(1)}, \beta_{l}^{(2)}, \beta_{l}^{(3)}\right)$ are the barycentric coordinates of the $s$-direction with respect to $T_{l}$;

- $\left(\gamma_{l}^{(1)}, \gamma_{l}^{(2)}, \gamma_{l}^{(3)}\right)$ are the barycentric coordinates of the $t$-direction with respect to $T_{l}$;

- at vertex $\mathbf{V}_{l}$ the basis function $B_{l}^{(j)}$ has a directional derivative equal to zero in the direction of the edge of $T_{l}$ opposite to $\mathbf{Q}_{l}^{(j)}$.

Finally, for each vertex $\mathbf{V}_{l}$ we define its PS points as the vertex itself and the midpoints of all the edges of the PS refinement $\mathcal{T}^{*}$ containing $\mathbf{V}_{l}$, see Fig. 3. From [5], Section 4, we have the following result (see also Figs. 3 and 4 ).

Theorem 1. The functions $B_{l}^{(j)}, j=1,2,3$, are nonnegative if and only if the PS triangle $T_{l}$ contains all the PS points associated to the vertex $\mathbf{V}_{l}$.

Summarizing, the PS B-splines associated to each vertex $\mathbf{V}_{l}$ of $\mathcal{T}$ are uniquely associated to the triple of points $\mathbf{Q}_{l}^{(j)}, j=1,2,3$, forming the PS triangle. Hence, PS triangles can be efficiently used to geometrically identify and describe the PS B-splines and their properties instead of $\alpha_{l}^{(j)}, \beta_{l}^{(j)}, \gamma_{l}^{(j)}$. PS triangles are not uniquely defined (see Fig. 3). One possibility for their construction is to compute a triangle of minimal area subject to the constraints of Theorem 1, see [5]. For an easier to implement but still satisfying alternative, we refer to $[25,26]$. Fig. 3 depicts two choices of PS triangles; the left PS triangle has minimal area.

Being equipped with a B-spline like basis, PS splines admit a straightforward rational extension as stated in the following Definition.

Definition 1. A NURPS (Non-Uniform Rational PS) surface on a PS refinement $\mathcal{T}^{*}$ of a triangulation $\mathcal{T}$ of $\Omega_{0}$ is defined as

$$
\mathbf{S}(s, t):=\sum_{i=1}^{N_{V}} \sum_{j=1}^{3} \mathbf{c}_{i}^{(j)} P_{i}^{(j)}(s, t), \quad P_{i}^{(j)}(s, t):=\frac{\omega_{i}^{(j)} B_{i}^{(j)}(s, t)}{\sum_{l=1}^{N_{V}} \sum_{r=1}^{3} \omega_{l}^{(r)} B_{l}^{(r)}(s, t)},
$$

where $\mathbf{c}_{i}^{(j)} \in \mathbb{R}^{d}$ are called NURPS control points, $B_{i}^{(j)}$ are the normalized PS $B$-splines, and $\omega_{i}^{(j)}$ are positive weights. 

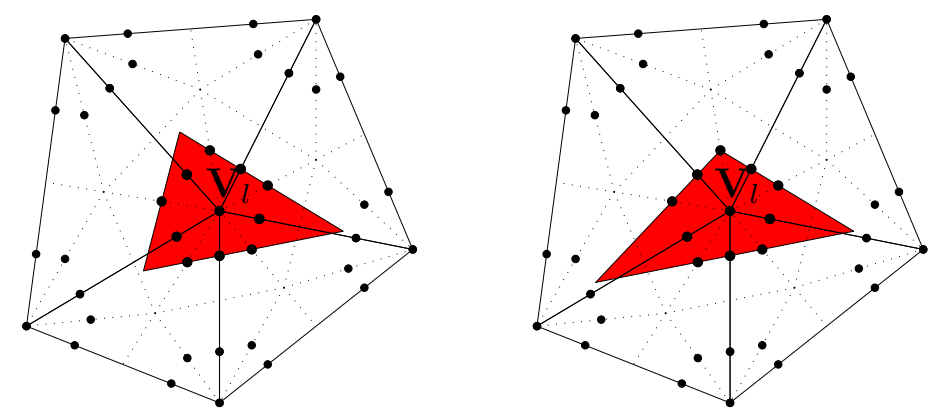

Figure 3: Location of the PS points (black bullets), and two possible "valid" PS triangles for vertex $\mathbf{V}_{l}$ (shaded), see Theorem 1. Left: PS triangle with minimal area. Right: a PS triangle of nearly minimal area.
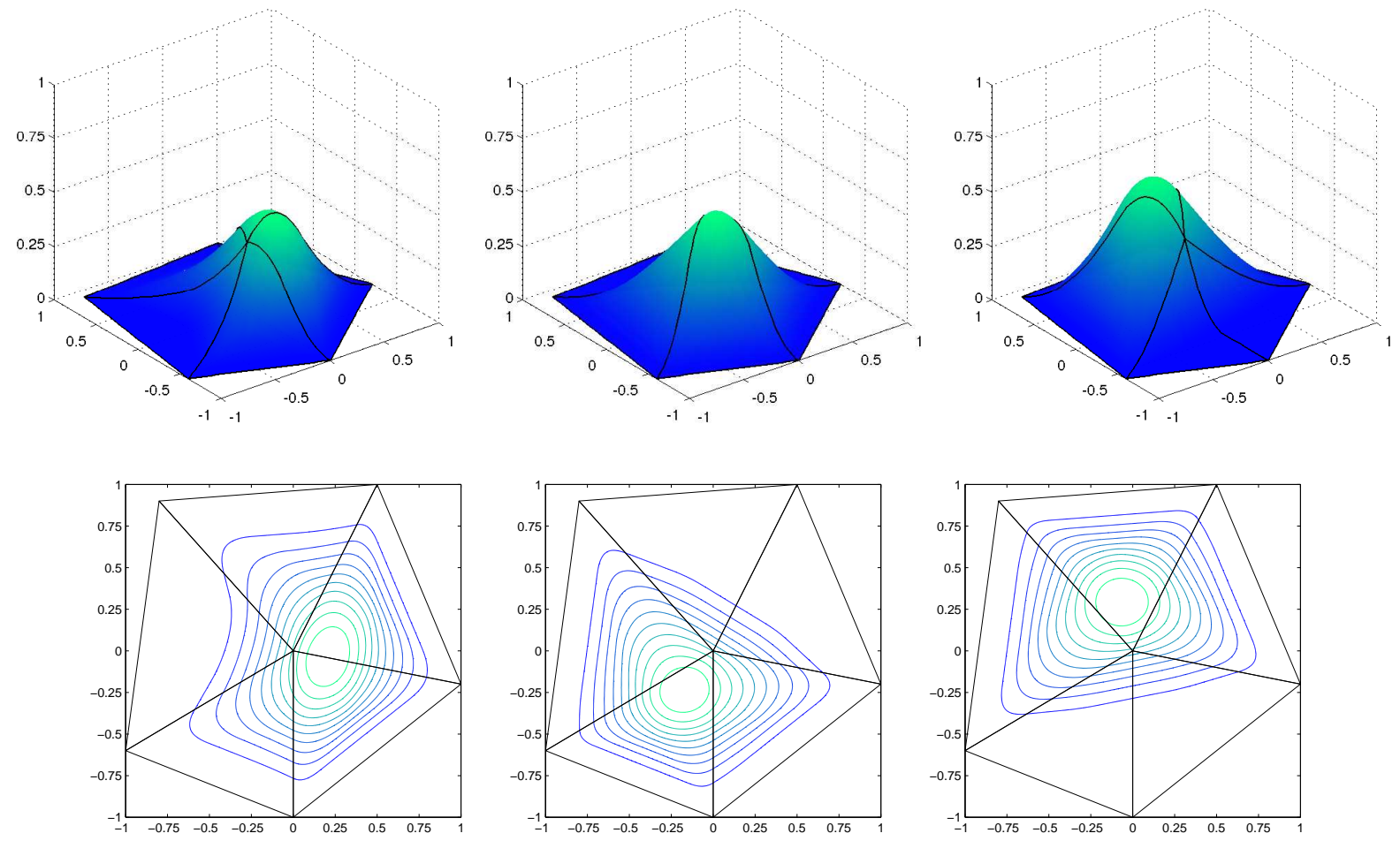

Figure 4: The three PS B-splines $B_{l}^{(j)}, j=1,2,3$, associated with the vertex $\mathbf{V}_{l}$ and the optimal PS triangle in Fig. 3 (left). Top: the functions $B_{l}^{(j)}$. Bottom: the contour lines of $B_{l}^{(j)}$. 
NURPS surfaces can be represented in a Bernstein-Bézier formulation by means of rational Bézier ordinates [29]. They can then be evaluated and manipulated in a stable way by using the rational de Casteljau algorithm. With particular choices for the control points and weights, NURPS can exactly represent patches on quadric surfaces. The influence of the weights on a NURPS surface can be described in a geometrically intuitive way [24].

As for classical NURBS, see (4), it is sometimes useful to consider the so-called homogeneous representation of (11), in which the NURPS surface is decoupled into $d+1$ standard PS spline components, i.e.

$$
\left(\sum_{i=1}^{N_{V}} \sum_{j=1}^{3} \mathbf{c}_{i}^{(j)} \omega_{i}^{(j)} B_{i}^{(j)}(s, t), \quad \sum_{i=1}^{N_{V}} \sum_{j=1}^{3} \omega_{i}^{(j)} B_{i}^{(j)}(s, t)\right) .
$$

\section{Conversion of a planar NURBS domain}

This section and the next one are the core of the paper. Here we provide an efficient procedure to describe in a NURPS form a planar domain represented by means of a tensor-product quadratic NURBS map, as in (6). As mentioned in the introduction, the interest of such a conversion is mainly motivated by the use of PS B-splines and their rational extension (NURPS) in $\operatorname{IgA}$.

From now on we assume that the knot sequences defining the NURBS patches are

$$
\begin{gathered}
0=u_{1}=u_{2}=u_{3}<\cdots<u_{n+1}=u_{n+2}=u_{n+3}=1, \\
0=\bar{u}_{1}=\bar{u}_{2}=\bar{u}_{3}<\cdots<\bar{u}_{m+1}=\bar{u}_{m+2}=\bar{u}_{m+3}=1 .
\end{gathered}
$$

We denote by $\Omega$ the image of the unit square $\Omega_{0}$ by a quadratic NURBS map defined by the knot sequences (12) with positive weights,

$$
\mathbf{G}(s, t):=\sum_{i=1}^{n} \sum_{j=1}^{m} \mathbf{p}_{i, j} R_{i, j}(s, t), \quad \mathbf{p}_{i, j} \in \mathbb{R}^{2},
$$

such that $\partial \Omega=\mathbf{G}\left(\partial \Omega_{0}\right)$.

Assuming the representation (13) is given, we want to determine a triangulation of $\Omega_{0}$, a PS refinement of it, PS B-splines, positive weights and NURPS control points, so that $\Omega$ can be seen as the image of a NURPS map $\mathbf{S}$ which 
exactly reproduces $\mathbf{G}$ on the boundary $\partial \Omega_{0}$, i.e.

$$
\mathbf{S}(s, t):=\sum_{i=1}^{N_{V}} \sum_{j=1}^{3} \mathbf{c}_{i}^{(j)} P_{i}^{(j)}(s, t), \quad \mathbf{c}_{i}^{(j)} \in \mathbb{R}^{2},
$$

satisfying

$$
\mathbf{G}\left(\Omega_{0}\right)=\left\{(x, y) \in \mathbb{R}^{2}:(x, y)=\mathbf{S}(s, t),(s, t) \in \Omega_{0}\right\},
$$

and

$$
\mathbf{G}(s, t)=\mathbf{S}(s, t), \quad(s, t) \in \partial \Omega_{0} .
$$

PS B-splines have a local structure and admit a local construction. Thus, along the boundary of $\Omega_{0}$, only PS B-splines associated to the boundary vertices of the triangulation $\mathcal{T}$ are of interest, because they are the only ones which can be non-zero. We will refer to them as boundary PS B-splines.

We can split the conversion process in the following two steps, which will be discussed in detail in the next two subsections.

i) Boundary reproduction: determine a triangulation $\mathcal{T}$ of $\Omega_{0}$, its PS refinement $\mathcal{T}^{*}$, boundary PS B-splines and corresponding positive weights and NURPS control points so that the NURBS map $\mathbf{G}$ is reproduced on $\partial \Omega_{0}$, i.e. (15) holds.

ii) Interior description: with the triangulation $\mathcal{T}$ of $\Omega_{0}$, its PS refinement $\mathcal{T}^{*}$, and boundary PS B-splines determined in the previous step, construct the remaining PS B-splines and compute the corresponding weights and NURPS control points so that (14) holds.

\subsection{Boundary reproduction}

In this subsection we describe an efficient procedure to obtain the reproduction of the geometry map $\mathbf{G}$ on $\partial \Omega_{0}$. Along the boundary, the twodimensional basis elements defined in (5) reduce to the onedimensional basis functions in (3). More precisely,

$$
\begin{array}{lll}
R_{k, 1}(s, 0)=R_{k, \mathbf{u}, \mathbf{W}_{1}}(s), & R_{k, m}(s, 1)=R_{k, \mathbf{u}, \mathbf{W}_{m}}(s), & k=1, \ldots, n, \\
R_{1, r}(0, t)=R_{r, \overline{\mathbf{u}}, \overline{\mathbf{w}}_{1}}(t), & R_{n, r}(1, t)=R_{r, \overline{\mathbf{u}}, \overline{\mathbf{W}}_{n}}(t), & r=1, \ldots, m,
\end{array}
$$

for suitable sequences of weights

$$
\mathbf{W}_{r}:=\left\{w_{1, r}, \ldots, w_{n, r}\right\}, \quad \overline{\mathbf{W}}_{k}:=\left\{w_{k, 1}, \ldots, w_{k, m}\right\}, \quad r=1, m, \quad k=1, n .
$$


Therefore, we construct a triangulation $\mathcal{T}$ of $\Omega_{0}$, its PS refinement $\mathcal{T}^{*}$, PS B-splines and positive weights and NURPS control points, such that

$$
\begin{aligned}
R_{k, \mathbf{u}, \mathbf{W}_{r}}(s) & =\sum_{i=1}^{N_{V}} \sum_{j=1}^{3} c_{i, k, r}^{(j)} P_{i}^{(j)}\left(s, \delta_{r, m}\right), \quad c_{i, k, r}^{(j)} \in \mathbb{R}, \quad k=1, \ldots, n, \quad r=1, m, \\
R_{r, \overline{\mathbf{u}}, \overline{\mathbf{W}}_{k}}(t) & =\sum_{i=1}^{N_{V}} \sum_{j=1}^{3} \bar{c}_{i, k, r}^{(j)} P_{i}^{(j)}\left(\delta_{k, n}, t\right), \quad \bar{c}_{i, k, r}^{(j)} \in \mathbb{R}, \quad k=1, n, \quad r=1, \ldots, m,
\end{aligned}
$$

where $\delta_{i, j}$ denotes Kronecker's symbol. Let us describe the procedure along the boundary edge $e_{1}:=\{(s, 0), s \in[0,1]\}$ of $\Omega_{0}$. The remaining boundary cases are completely similar. Since PS B-splines have a local structure and admit a local construction, it suffices to consider PS B-splines corresponding to vertices of the triangulation belonging to such an edge. We proceed as follows.

- Choose a triangulation of $\Omega_{0}$ such that the boundary vertices $\mathbf{V}_{i}, i=$ $1, \ldots, n-1$, belonging to the edge $e_{1}$ are at the knot positions, i.e.,

$$
\mathbf{V}_{i}:=\left(u_{i+2}, 0\right), \quad i=1, \ldots, n-1 .
$$

- Choose the PS refinement on the boundary of $\Omega_{0}$ selecting a point $\left(z_{i}, 0\right)$, for each boundary edge $\mathbf{V}_{i} \mathbf{V}_{i+1}, i=1, \ldots, n-2$.

- Define the PS B-splines related to these boundary vertices - which do not vanish along the edge - by choosing their PS triangles as follows (only two points of each PS triangle are fixed):

$$
\begin{array}{ll}
\mathbf{Q}_{1}^{(1)}:=\mathbf{V}_{1}, & \mathbf{Q}_{1}^{(2)}:=\left(\mathbf{V}_{1}+\mathbf{V}_{2}\right) / 2, \\
\mathbf{Q}_{i}^{(1)}:=\left(\mathbf{V}_{i-1}+\mathbf{V}_{i}\right) / 2, & \mathbf{Q}_{i}^{(2)}:=\left(\mathbf{V}_{i}+\mathbf{V}_{i+1}\right) / 2, \quad i=2, \ldots, n-2, \\
\mathbf{Q}_{n-1}^{(1)}:=\left(\mathbf{V}_{n-2}+\mathbf{V}_{n-1}\right) / 2, & \mathbf{Q}_{n-1}^{(2)}:=\mathbf{V}_{n-1} .
\end{array}
$$

We then arrive at the following proposition, see also Fig. 5.

Proposition 1. With the choices (17) and (18) we have

$$
\begin{aligned}
& B_{i}^{(3)}(s, 0)=0, \quad i=1, \ldots, n-1, \\
& N_{1, \mathbf{u}}(s)=B_{1}^{(1)}(s, 0), \\
& N_{i, \mathbf{u}}(s)=B_{i-1}^{(2)}(s, 0)+B_{i}^{(1)}(s, 0), \quad i=2, \ldots, n-1, \\
& N_{n, \mathbf{u}}(s)=B_{n-1}^{(2)}(s, 0) .
\end{aligned}
$$



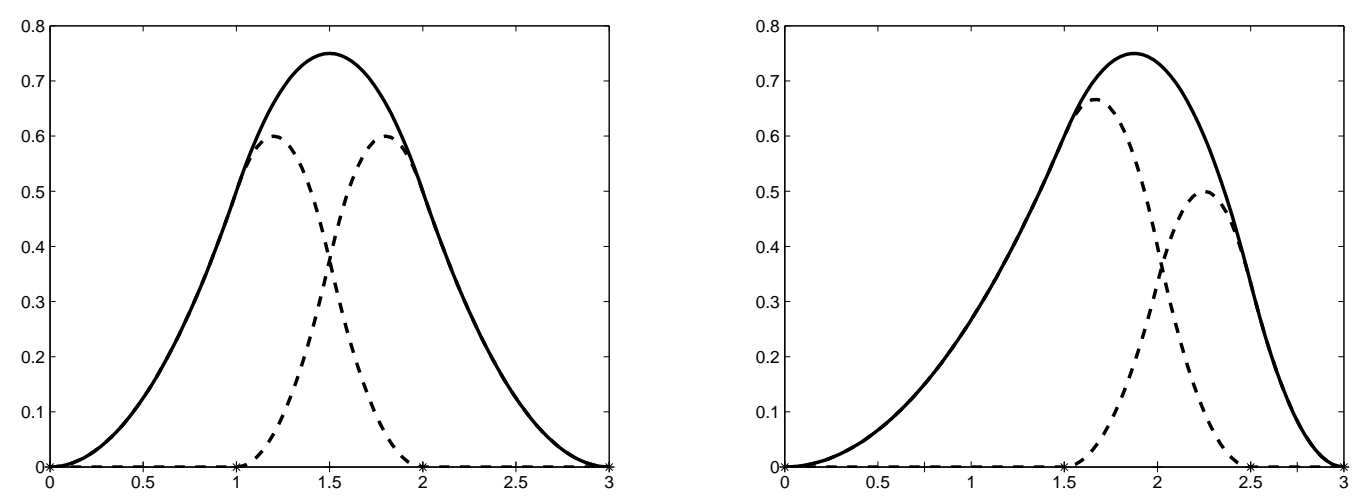

Figure 5: The quadratic B-spline $N_{i, \mathbf{u}}(s)$ and the PS B-splines $B_{i-1}^{(2)}(s, 0), B_{i}^{(1)}(s, 0)$ (dashed lines) for different knot sequences. Left: [ $\left[\begin{array}{llll}0 & 1 & 2 & 3\end{array}\right]$. Right: [ [ $\left.\begin{array}{lllll}0 & 1.5 & 2.5 & 3\end{array}\right]$. In both cases $z_{i}$ are selected as the midpoints of the corresponding edges.

Proof. Each $B_{i}^{(j)}$ is a $C^{1}$ piecewise quadratic defined over the knot sequence

$$
\begin{array}{ll}
\xi_{l-1}:=u_{l}, & l=1,2,3 \\
\xi_{2 l+1}:=z_{l}, & l=1, \ldots, n-2, \\
\xi_{2 l}:=u_{l+2}, & l=2, \ldots, n-2, \\
\xi_{l+n-3}:=u_{l}, & l=n+1, n+2, n+3,
\end{array}
$$

and has its support in $\left[u_{i+1}, u_{i+3}\right]=\left[\xi_{2 i-2}, \xi_{2 i+2}\right]$. From (10) we obtain that ${ }^{5}$

$$
\begin{gathered}
\alpha_{1}^{(1)}=1, \alpha_{1}^{(2)}=\alpha_{1}^{(3)}=0, \beta_{1}^{(1)}=-\frac{2}{u_{4}-u_{3}}, \beta_{1}^{(2)}=-\beta_{1}^{(1)}, \beta_{1}^{(3)}=0, \\
\alpha_{n-1}^{(2)}=1, \alpha_{n-1}^{(1)}=\alpha_{n-1}^{(3)}=0, \beta_{n-1}^{(2)}=\frac{2}{u_{n}-u_{n+1}}, \beta_{n-1}^{(1)}=-\beta_{n-1}^{(2)}, \beta_{n-1}^{(3)}=0,
\end{gathered}
$$

and, for $i=2, \ldots, n-2$,

$$
\begin{array}{lll}
\alpha_{i}^{(1)}=\frac{u_{i+3}-u_{i+2}}{u_{i+3}-u_{i+1}}, & \alpha_{i}^{(2)}=1-\alpha_{i}^{(1)}, & \alpha_{i}^{(3)}=0, \\
\beta_{i}^{(1)}=-\frac{2}{u_{i+3}-u_{i+1}}, & \beta_{i}^{(2)}=-\beta_{i}^{(1)}, & \beta_{i}^{(3)}=0 .
\end{array}
$$

Therefore $B_{i}^{(3)}(s, 0)=0$, because it is a $C^{1}$ piecewise quadratic with value zero and vanishing first derivatives at the knots.

\footnotetext{
${ }^{5} \mathrm{By}(18)$, the behavior of the considered boundary PS B-splines along $e_{1}$ is independent of the choice of the third vertices of the PS triangles.
} 
On the other hand, from (1) we know that

$$
N_{i, \mathbf{u}}(s)= \begin{cases}\frac{\left(s-u_{i}\right)^{2}}{\left(u_{i+2}-u_{i}\right)\left(u_{i+1}-u_{i}\right)}, & \text { if } s \in\left[u_{i}, u_{i+1}\right), \\ \frac{\left(u_{i+2}-s\right)\left(s-u_{i}\right)}{\left(u_{i+2}-u_{i}\right)\left(u_{i+2}-u_{i+1}\right)}+\frac{\left(s-u_{i+1}\right)\left(u_{i+3}-s\right)}{\left(u_{i+3}-u_{i+1}\right)\left(u_{i+2}-u_{i+1}\right)}, & \text { if } s \in\left[u_{i+1}, u_{i+2}\right), \\ \frac{\left(u_{i+3}-s\right)^{2}}{\left(u_{i+3}-u_{i+1}\right)\left(u_{i+3}-u_{i+2}\right)}, & \text { if } s \in\left[u_{i+2}, u_{i+3}\right), \\ 0, & \text { elsewhere. }\end{cases}
$$

With elementary computations we obtain that the left and right sides of (19) and their first derivatives agree at the knots $\mathbf{u}$. Therefore, (19) holds for $s \in[0,1]$, because $N_{r, \mathbf{u}}(s)$ and $B_{i}^{(j)}(s, 0), j=1,2$, are uniquely determined for $s \in[0,1]$ by their values and derivatives at the knots $\mathbf{u}$.

From Proposition 1 we immediately obtain the following result.

Proposition 2. Let the set of rational piecewise quadratic functions

$$
\left\{R_{i, \mathbf{u}, \mathbf{W}_{1}}, i=1, \ldots, n\right\}
$$

in (16) be given. Then

$$
\begin{aligned}
& R_{1, \mathbf{u}, \mathbf{W}_{1}}(s)=P_{1}^{(1)}(s, 0), \\
& R_{i, \mathbf{u}, \mathbf{W}_{1}}(s)=P_{i-1}^{(2)}(s, 0)+P_{i}^{(1)}(s, 0), \\
& R_{n, \mathbf{u}, \mathbf{W}_{1}}(s)=P_{n-1}^{(2)}(s, 0),
\end{aligned}
$$

where, for $i=1, \ldots, n-1, j=1,2$,

$$
P_{i}^{(j)}(s, 0)=\frac{\omega_{i}^{(j)} B_{i}^{(j)}(s, 0)}{\sum_{l=1}^{n-1} \sum_{r=1}^{2} \omega_{l}^{(r)} B_{l}^{(r)}(s, 0)},
$$

$B_{i}^{(j)}, i=1, \ldots, n-1, j=1,2$ are the PS B-splines defined by (17) and (18) and

$$
\begin{aligned}
& \omega_{1}^{(1)}=w_{1,1}, \\
& \omega_{i}^{(1)}=\omega_{i-1}^{(2)}=w_{i, 1}, \quad i=2, \ldots, n-1, \\
& \omega_{n-1}^{(2)}=w_{n, 1} .
\end{aligned}
$$


Moreover, for any curve $\mathbf{C}^{\mathbf{W}_{1}}(s):=\sum_{l=1}^{n} \mathbf{p}_{l} R_{l, \mathbf{u}, \mathbf{W}_{1}}(s)$ we have

$$
\mathbf{C}^{\mathbf{W}_{1}}(s)=\sum_{i=1}^{n-1} \sum_{j=1}^{2} \mathbf{c}_{i}^{(j)} P_{i}^{(j)}(s, 0)
$$

where

$$
\begin{aligned}
& \mathbf{c}_{1}^{(1)}=\mathbf{p}_{1}, \\
& \mathbf{c}_{i}^{(1)}=\mathbf{c}_{i-1}^{(2)}=\mathbf{p}_{i}, \quad i=2, \ldots, n-1, \\
& \mathbf{c}_{n-1}^{(2)}=\mathbf{p}_{n} .
\end{aligned}
$$

Proposition 2 implies that $\mathbf{G}$ can be exactly reproduced on the boundary by a NURPS representation involving positive weights and nonnegative basis functions, when the PS triangles along the boundary are chosen such that both (18) and Theorem 1 are satisfied.

\subsection{Interior description}

Due to the local construction of PS B-splines, the boundary reproduction of the map $\mathbf{G}$ discussed in the previous subsection basically results in determining the boundary vertices of a triangulation of $\Omega_{0}$, some related PS B-splines and corresponding weights and NURPS control points.

Now, assuming the triangulation $\mathcal{T}$ and its PS refinement have been fixed, we determine PS B-splines, weights and NURPS control points to construct the NURPS map in (14) in the interior of $\Omega_{0}$.

To this end, let us consider both the NURBS and the NURPS maps in their homogeneous forms, i.e.

$$
\begin{gathered}
\left(\sum_{i=1}^{n} \sum_{j=1}^{m} \mathbf{p}_{i, j} w_{i, j} N_{i, \mathbf{u}}(s) N_{j, \overline{\mathbf{u}}}(t), \quad \sum_{i=1}^{n} \sum_{j=1}^{m} w_{i, j} N_{i, \mathbf{u}}(s) N_{j, \overline{\mathbf{u}}}(t)\right) \\
=:\left(X_{N}(s, t), Y_{N}(s, t), D_{N}(s, t)\right), \\
\left(\sum_{i=1}^{N_{V}} \sum_{j=1}^{3} \mathbf{c}_{i}^{(j)} \omega_{i}^{(j)} B_{i}^{(j)}(s, t), \quad \sum_{i=1}^{N_{V}} \sum_{j=1}^{3} \omega_{i}^{(j)} B_{i}^{(j)}(s, t)\right) \\
=:\left(X_{P S}(s, t), Y_{P S}(s, t), D_{P S}(s, t)\right) .
\end{gathered}
$$

First we construct a set of PS B-splines on the considered PS refinement of $\mathcal{T}$ associated to interior vertices by considering PS triangles of nearly minimal 
area as described in [25].

Then, we may determine the NURPS weights $\omega_{i}^{(j)}$ by Hermite interpolation at the vertices of the last component, $D_{N}$, of the homogeneous form of the NURBS map (20) in the given PS spline space. Once the weights are fixed, we may compute the control points $\mathbf{c}_{i}^{(j)}$ by Hermite interpolation of the first two components of (20) in the same PS spline space.

Although the above procedure usually provides satisfying results in practice, we remark that it does not guarantee that the computed weights $\omega_{i}^{(j)}$ are always positive, even if the NURBS weights $w_{i, j}$ are.

From [5] we know that, for a given vertex $\mathbf{V}_{l}=\left(s_{l}, t_{l}\right)$, the corresponding weights $\omega_{l}^{(j)}$ are so that its control triangle, i.e. the triangle with vertices

$$
\left(Q_{l, s}^{(j)}, Q_{l, t}^{(j)}, \omega_{l}^{(j)}\right), \quad j=1,2,3,
$$

is tangent to the surface $D_{N}$ at $\mathbf{V}_{l}$. If positive weights are considered in the NURBS map, then $D_{N}$ is positive in $\Omega_{0}$. Therefore, in the case that negative or zero values are obtained for $\omega_{i}^{(j)}$ by the Hermite interpolation process, we can apply a correction step using a shape-preserving interpolation strategy. Such a shape-preserving scheme basically consists of reducing the moduli of the partial derivatives of $D_{N}$ at $\mathbf{V}_{l}$. We point out that this shape-preserving correction is not needed when the triangulation is fine enough. Indeed, when the triangulation is refined, the Powell-Sabin Hermite interpolant approaches $D_{N}$, which is positive. Therefore, the Powell-Sabin interpolant will be positive as well.

In general, it is not guaranteed that the above strategy provides a NURPS map of which the image of $\Omega_{0}$ is exactly the domain $\Omega$, i.e. (14) holds. Nevertheless, in practical situations the right domain is obtained by this strategy. Moreover, when refining the triangulation of $\Omega_{0}$, the image of the NURPS map will always converge to the one of the given NURBS transformation. This is also illustrated in Section 6.

We end this section by observing that this procedure provides at least a third order accurate NURPS approximation of the given NURBS parametrization of $\Omega$. This can be shown as follows. We assume that the triangulation of the NURPS map is chosen such that the grid lines of the tensor-product mesh of the NURBS map are situated on edges in the triangulation. Then, for instance, for the first component we have

$$
\frac{X_{N}}{D_{N}}-\frac{X_{P S}}{D_{P S}}=\frac{X_{N}\left(D_{P S}-D_{N}\right)+D_{N}\left(X_{N}-X_{P S}\right)}{D_{N} D_{P S}} .
$$


To bound the above error, it is sufficient to consider it in any element of the tensor-product NURBS mesh, because the construction of the PowellSabin Hermite interpolant is local. In such an element, the components of the NURBS map are smooth enough, so that $D_{P S}$ and $X_{P S}$ provide a third order approximation to $D_{N}$ and $X_{N}$, respectively, see [20]. Moreover, $D_{N}$ is bounded away from zero by assumption, and therefore the same holds true for $D_{P S}$ when the triangulation is fine enough.

Finally, we note that the procedure described in the two previous subsections works componentwise and therefore it can be immediately extended to the multi-dimensional setting, see Subsection 6.2. The same applies to the error estimate discussed above.

\subsection{Positivity of constructed PS B-splines and related weights}

A main point in the conversion process is that the used PS B-splines have to be nonnegative and the corresponding weights positive. According to Subsection 4.2 this can be always achieved for PS B-splines associated to interior vertices of $\mathcal{T}$.

As far as boundary vertices are concerned, Proposition 2 ensures positivity of weights. However, for any triangulation satisfying (17) with a given PS refinement, it is not always possible to find a set of PS triangles related to the boundary vertices, such that (18) holds and such that Theorem 1 is satisfied to guarantee the nonnegativity of the PS B-splines, see Fig. 6. In this subsection, we will show that both types of constraints on the boundary PS triangles can be satisfied under some mild assumption on $\mathcal{T}$.

For notational convenience, let us put

$$
\Omega_{0, l}^{1 / 2}:=\left\{\mathbf{X} \in \Omega_{0, l}: \mathbf{V}_{l}+2\left(\mathbf{X}-\mathbf{V}_{l}\right) \in \Omega_{0, l}\right\}
$$

We will refer to the set $\Omega_{0, l}^{1 / 2}$ as the half molecule associated to $\mathbf{V}_{l}$, see Fig. 7 . Of course, if a PS triangle contains the half molecule of the vertex, then the conditions of Theorem 1 are satisfied. Therefore, we state the following definition, see Fig. 7.

Definition 2. A triangulation $\mathcal{T}$ of the unit square $\Omega_{0}$ is admissible if it is possible to construct PS B-splines associated to boundary vertices according to (17) and (18), so that the following hypothesis holds:

(H1) for each boundary vertex the corresponding PS triangle contains the half molecule associated to the vertex itself. 

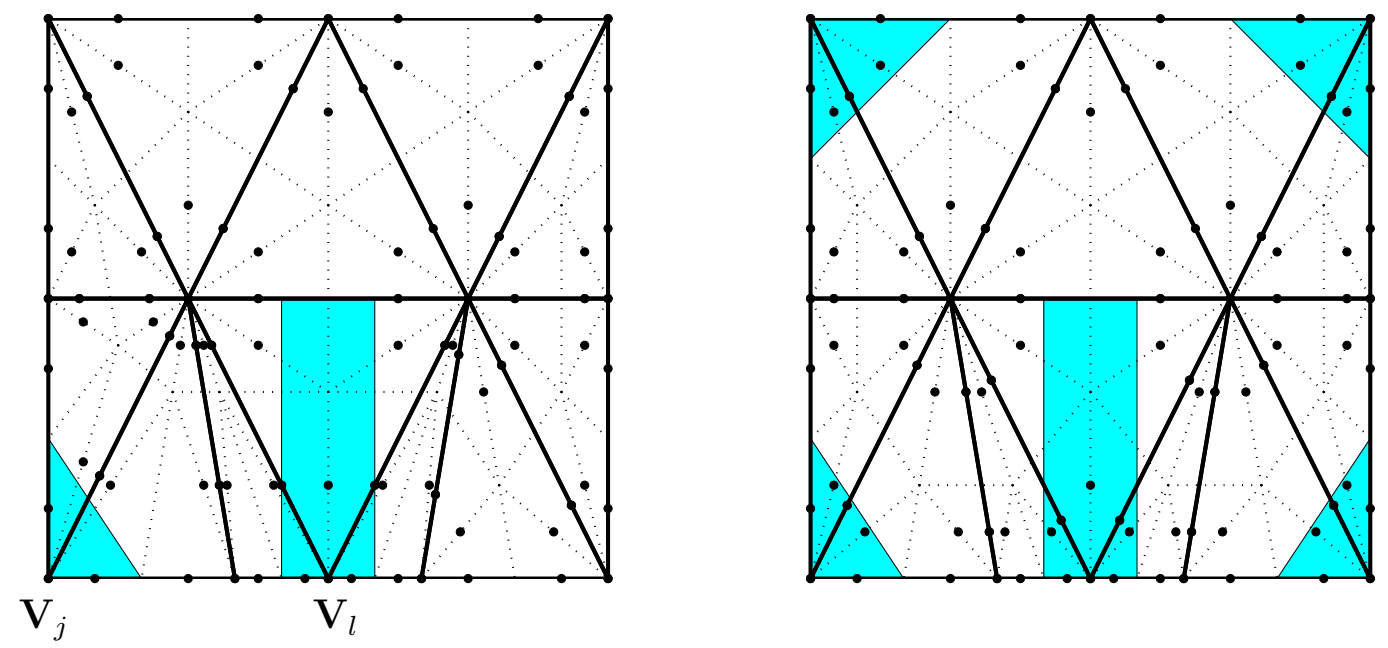

Figure 6: Two PS refinements for the same triangulation. Left: it is not possible to satisfy the conditions in Theorem 1 for vertices $\mathbf{V}_{j}$ and $\mathbf{V}_{l}$ if the corresponding PS triangles are constructed according to (18). Right: the conditions in Theorem 1 can be satisfied for all boundary vertices with PS triangles constructed according to (18). The shaded area at the corners depicts the PS triangle defined by (18). For $\mathbf{V}_{l}$, if there are PS points outside the shaded area at both sides then it is not possible to construct a PS triangle ensuring nonnegativity.
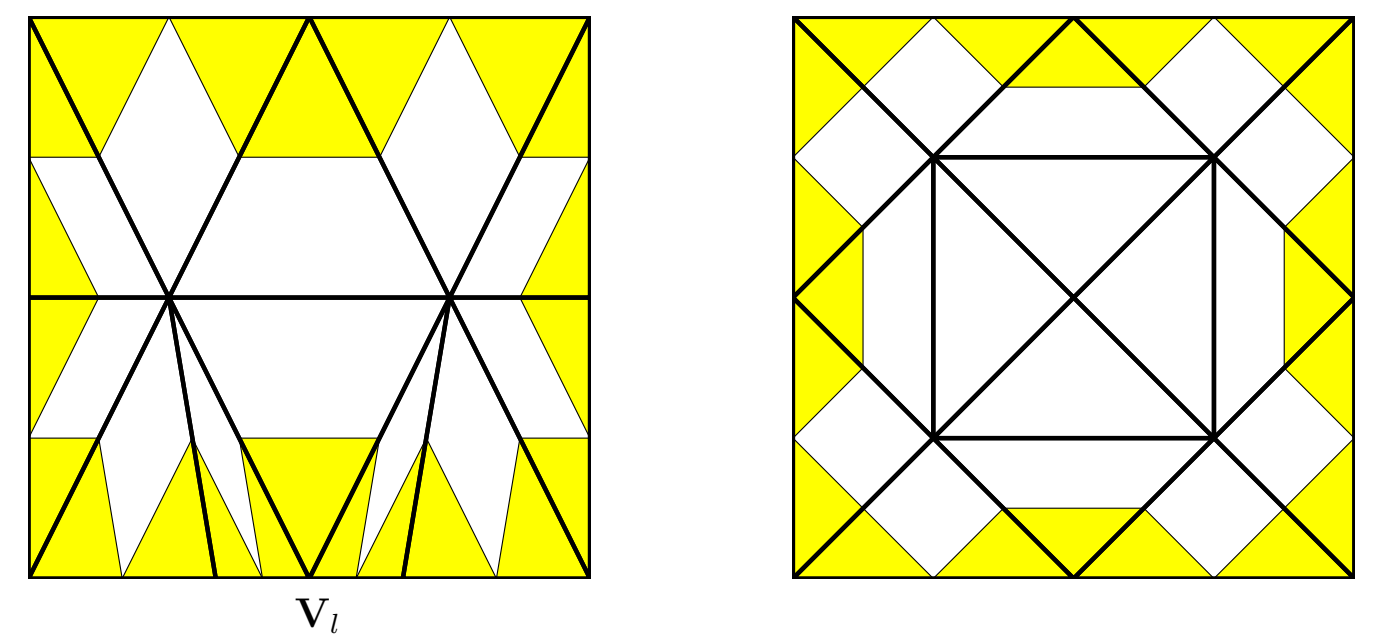

Figure 7: Left: no admissible triangulation of the unit square. The PS B-splines associated to the vertex $\mathbf{V}_{l}$ and to the four corners constructed according to (17) and (18) cannot satisfy (H1). Right: admissible triangulation of the unit square. In both cases the half molecules of boundary vertices are shaded. 
Remark 1. To be admissible is a property of the triangulation. It is independent of the chosen PS refinement. Any four directional triangulation of the unit square is admissible, see for example Fig. 11.

The next proposition ensures nonnegativity of PS B-splines used in our conversion.

Proposition 3. Let $\mathbf{G}$ be a quadratic NURBS map as in (13). Let $\mathcal{T}$ be an admissible triangulation of $\Omega_{0}$ with a given $P S$ refinement. Then we can construct a set of nonnegative PS B-splines

$$
\left\{B_{i}^{(j)}, i=1, \ldots, N_{V}, j=1,2,3\right\}
$$

so that

$$
\mathbf{G}(s, t)=\sum_{i=1}^{N_{V}} \sum_{j=1}^{3} \mathbf{c}_{i}^{(j)} P_{i}^{(j)}(s, t), \quad(s, t) \in \partial \Omega_{0},
$$

where

$$
P_{i}^{(j)}(s, t):=\frac{\omega_{i}^{(j)} B_{i}^{(j)}(s, t)}{\sum_{l=1}^{N_{V}} \sum_{r=1}^{3} \omega_{l}^{(r)} B_{l}^{(r)}(s, t)}, \quad \omega_{i}^{(j)}>0 .
$$

Proof. From Subsection 4.2 we know that only boundary PS B-splines and corresponding weights have to be considered. Let the boundary PS B-splines in (24) be determined according to (17) and (18). From Proposition 2 we have that (25) is satisfied with positive weights. Since $\mathcal{T}$ is admissible, (H1) ensures that the PS B-splines are nonnegative.

Note that to deal with an admissible triangulation is just a sufficient condition to ensure that the thesis in Proposition 3 holds, because nonnegativity of the constructed PS B-splines strongly depends on the chosen PS refinement, see both Figs. 6 and 7. Nevertheless, in practice it is very useful to have a condition which can be easily checked and which is independent of the PS refinement.

Moreover, we remark that even if nonnegativity of boundary PS B-splines could be obtained for more general triangulations, assuming $\mathcal{T}$ is admissible ensures that such a crucial property can be kept along any local refinement of $\mathcal{T}$. This will be addressed in the following section. 


\section{Refining the given triangulation}

In view of applications in IgA, in this section we focus on the construction of NURPS representations of the (planar) domain $\Omega$ defined over locally refined triangulations of the parameter domain $\Omega_{0}$. More precisely, assuming a refinement $\widehat{\mathcal{T}}$ of the initial triangulation $\mathcal{T}$ is given, we want to construct nonnegative PS B-splines, positive weights, and control points, so that the given NURBS map is reproduced on the boundary of $\Omega_{0}$. In contrast to the previous section, the triangulation $\widehat{\mathcal{T}}$ is now given and we cannot make any assumption on it, but it is a refinement of an admissible triangulation of $\Omega_{0}$. Along the entire refinement process, it is essential to maintain nonnegativity of the PS B-splines as well as positivity of the weights in the NURPS representations. Due to their local support, PS B-splines associated to interior vertices do not affect the boundary of $\Omega_{0}$ and so of $\Omega$. In the next subsections, we analyze the nonnegativity of boundary PS B-splines along the refinement process, both on the boundary and in the interior of $\Omega_{0}$, keeping in mind that (the rational extension of) these PS B-splines must reproduce the boundary of $\Omega$ as given by the NURBS map using positive weights.

\subsection{Analyzing boundary $P S$ B-splines on $\partial \Omega_{0}$}

For the sake of simplicity, we limit ourselves to a single edge of $\Omega_{0}$, say $e_{1}$. Let $\mathcal{V}_{1}$ be a set of boundary vertices along $e_{1}$ as in (17), and let $\widehat{\mathcal{V}}_{1}$ be another set of boundary vertices associated in a similar way to a different sequence of knots $\widehat{\mathbf{u}}$, that is

$$
\widehat{\mathcal{V}}_{1}=\left\{\widehat{\mathbf{V}}_{i}:=\left(\widehat{u}_{i+2}, 0\right), i=1, \ldots, \widehat{n}-1\right\},
$$

where

$$
0=\widehat{u}_{1}=\widehat{u}_{2}=\widehat{u}_{3}<\cdots<\widehat{u}_{\widehat{n}+1}=\widehat{u}_{\widehat{n}+2}=\widehat{u}_{\widehat{n}+3}=1 .
$$

Proposition 4. If $\mathbf{u} \subset \widehat{\mathbf{u}}$, then we can construct a set of boundary PSBsplines $\left\{\widehat{B}_{i}^{(j)}, i=1, \ldots, \widehat{n}-1, j=1,2\right\}$ associated to $\widehat{\mathcal{V}}_{1}$, so that for the NURBS map $\mathbf{G}$ in (13) we have

$$
\mathbf{G}(s, 0)=\sum_{i=1}^{\widehat{n}-1} \sum_{j=1}^{2} \widehat{\mathbf{c}}_{i}^{(j)} \widehat{P}_{i}^{(j)}(s, 0),
$$

where, for $i=1, \ldots, \widehat{n}-1, j=1,2$,

$$
\widehat{P}_{i}^{(j)}(s, 0)=\frac{\widehat{\omega}_{i}^{(j)} \widehat{B}_{i}^{(j)}(s, 0)}{\sum_{l=1}^{\widehat{n}-1} \sum_{r=1}^{2} \widehat{\omega}_{l}^{(r)} \widehat{B}_{l}^{(r)}(s, 0)}, \quad \widehat{\omega}_{i}^{(j)}>0 .
$$


Proof. Along $e_{1}$, the NURBS map $\mathbf{G}$ can be seen as the projection of a suitable B-spline curve in homogeneous form with three components in $\mathbb{S}_{2, \mathbf{u}}$ (see (4)). Since $\mathbf{u} \subset \widehat{\mathbf{u}}$, the well-known knot-insertion process, see e.g. [3], ensures that the coefficients of any element of $\mathbb{S}_{2, \mathbf{u}}$ expressed with respect to the B-spline basis in $\mathbb{S}_{2, \widehat{\mathbf{u}}}$ are obtained by convex combinations of the coefficients with respect to the B-spline basis in $\mathbb{S}_{2, \mathbf{u}}$. Because the weights in a NURBS representation of a planar curve can be regarded as the coefficients of the third component of the homogeneous form of the curve, any NURBS representation with positive weights related to the knot sequence $\mathbf{u}$, can be expressed in a NURBS representation with positive weights related to the knot sequence $\widehat{\mathbf{u}}$.

Let $\left\{\widehat{B}_{i}^{(j)}, i=1, \ldots, \widehat{n}-1, j=1,2,3\right\}$ be a set of PS B-splines associated to the vertices in $\widehat{\mathcal{V}}_{1}$ constructed according to $(17)-(18)$, replacing $\mathcal{V}_{1}$ by $\widehat{\mathcal{V}}_{1}$. Then Proposition 2 can be invoked to obtain (27), which guarantees positivity of weights in the NURPS representation.

We note that the PS B-splines considered in the proof of Proposition 4 are nonnegative along $e_{1}$. However, nonnegativity is not necessarily ensured in the interior of $\Omega_{0}$, because we do not know if it is possible to find a PS triangle for vertices in $\widehat{\mathcal{V}}_{1}$ such that (18) holds and Theorem 1 is satisfied, see Subsection 4.3.

To address the behavior of boundary PS B-splines constructed along the refinement process, the following property of boundary PS B-splines associated to different sequences of knots is of interest.

Proposition 5. Let $\left\{B_{k}^{(j)}, k=1, \ldots, n-1, j=1,2,3\right\}$ and $\left\{\widehat{B}_{i}^{(j)}, i=\right.$ $1, \ldots, \widehat{n}-1, j=1,2,3\}$ be $P S$ B-splines associated to two sets of boundary vertices as in (17) and (26) respectively. Let $\mathbf{Q}_{k}^{(j)}, \widehat{\mathbf{Q}}_{i}^{(j)}, j=1,2,3$, denote the vertices of the corresponding $P S$ triangles so that $\mathbf{Q}_{k}^{(j)}, \widehat{\mathbf{Q}}_{i}^{(j)}, j=1,2$, belong to the edge $e_{1}$. Let the function

$$
f(s)=\sum_{k=1}^{n-1} \sum_{j=1}^{2} \sigma_{k}^{(j)} B_{k}^{(j)}(s, 0)=\sum_{i=1}^{\widehat{n}-1} \sum_{j=1}^{2} \widehat{\sigma}_{i}^{(j)} \widehat{B}_{i}^{(j)}(s, 0), \quad s \in[0,1],
$$

be given. For any $i, k$ such that

$$
\widehat{\mathbf{V}}_{i}=\mathbf{V}_{k}, \text { and } \widehat{\mathbf{Q}}_{i}^{(j)}=\mathbf{Q}_{k}^{(j)}, j=1,2,
$$

we have

$$
\widehat{\sigma}_{i}^{(j)}=\sigma_{k}^{(j)}, j=1,2 .
$$


Proof. Along edge $e_{1}$, we have $B_{k}^{(3)}(s, 0)=\widehat{B}_{i}^{(3)}(s, 0)=0, k=1, \ldots, n-1$, $i=1, \ldots, \widehat{n}-1$. Moreover, from (10) we know that the values of $B_{k}^{(j)}$ and $\widehat{B}_{i}^{(j)}$ and of their derivatives along $e_{1}$ at the vertices do not depend on $\mathbf{Q}_{k}^{(3)}, \widehat{\mathbf{Q}}_{i}^{(3)}$. The values $\sigma_{k}^{(j)}\left(\widehat{\sigma}_{i}^{(j)}\right), j=1,2$, are completely specified by the values and the first derivatives of $f$ at the knots $\mathbf{u}(\widehat{\mathbf{u}})$. More precisely, in view of (8) and $(9), \sigma_{k}^{(j)}$ can be locally computed by solving a $2 \times 2$ linear system whose coefficients are $\alpha_{k}^{(j)}, \beta_{k}^{(j)}, j=1,2$. In the same way $\widehat{\sigma}_{i}^{(j)}$ depends on $\widehat{\alpha}_{i}^{(j)}, \widehat{\beta}_{i}^{(j)}$, $j=1,2$. Thus,

$$
\left(\begin{array}{c}
f\left(u_{k+2}\right) \\
\frac{\partial}{\partial s} f\left(u_{k+2}\right)
\end{array}\right)=\left(\begin{array}{cc}
\alpha_{k}^{(1)} & \alpha_{k}^{(2)} \\
\beta_{k}^{(1)} & \beta_{k}^{(2)}
\end{array}\right)\left(\begin{array}{c}
\sigma_{k}^{(1)} \\
\sigma_{k}^{(2)}
\end{array}\right)=\left(\begin{array}{cc}
\widehat{\alpha}_{i}^{(1)} & \widehat{\alpha}_{i}^{(2)} \\
\widehat{\beta}_{i}^{(1)} & \widehat{\beta}_{i}^{(2)}
\end{array}\right)\left(\begin{array}{c}
\widehat{\sigma}_{i}^{(1)} \\
\widehat{\sigma}_{i}^{(2)}
\end{array}\right) .
$$

In addition, from (28) and (10) we obtain that

$$
\widehat{\alpha}_{i}^{(j)}=\alpha_{k}^{(j)}, \quad \widehat{\beta}_{i}^{(j)}=\beta_{k}^{(j)}, \quad j=1,2,
$$

which implies (29).

\subsection{Analyzing boundary PS B-splines in $\Omega_{0}$}

Assuming $\mathcal{T}$ is an admissible triangulation, in the following we prove that for any refinement $\widehat{\mathcal{T}}$ of $\mathcal{T}$ it is possible to construct a suitable family of boundary PS B-splines, which are nonnegative and associated to positive weights when used to reproduce the given NURBS geometry map in (13) on $\partial \Omega_{0}$.

We assume that the vertices of $\mathcal{T}$ are given as in (7) and that

$$
\widehat{\mathbf{V}}_{l}, \quad l=1, \ldots, \widehat{N}_{V},
$$

are the vertices of $\widehat{\mathcal{T}}$. Moreover, we denote by $\mathcal{V}(\widehat{\mathcal{V}})$ the set of boundary vertices of $\mathcal{T}(\widehat{\mathcal{T}})$.

Proposition 6. Let a triangulation $\mathcal{T}$ of $\Omega_{0}$ be given equipped with a PS refinement and a collection of nonnegative $P S$ B-splines,

$$
\left\{B_{i}^{(j)}, i=1, \ldots, N_{V}, j=1,2,3\right\},
$$

so that (H1) is satisfied. Let $\widehat{\mathcal{T}}$ be a refinement of $\mathcal{T}$ equipped with a PS refinement, and let

$$
\left\{\widehat{B}_{i}^{(j)}, i=1, \ldots, \widehat{N}_{V}, j=1,2,3\right\}
$$


be a family of PS B-splines so that for each $\widehat{\mathbf{V}}_{i} \in \widehat{\mathcal{V}}$

$$
\begin{aligned}
& \text { if } \widehat{\mathbf{V}}_{i}=\mathbf{V}_{k} \in \mathcal{V}, \text { then } \widehat{\mathbf{Q}}_{i}^{(j)}:=\mathbf{Q}_{k}^{(j)}, j=1,2,3, \\
& \text { if } \widehat{\mathbf{V}}_{i} \notin \mathcal{V}, \text { then } \widehat{\mathbf{Q}}_{i}^{(j)}:=\frac{\mathbf{V}_{i_{j}}+\widehat{\mathbf{V}}_{i}}{2}, j=1,2,3,
\end{aligned}
$$

where $\mathbf{V}_{i_{1}} \mathbf{V}_{i_{2}} \mathbf{V}_{i_{3}}$ is the triangle of $\mathcal{T}$ such that $\widehat{\mathbf{V}}_{i}$ belongs to the (boundary) edge $\mathbf{V}_{i_{1}} \mathbf{V}_{i_{2}}$. Then, $\widehat{\mathcal{T}}$ satisfies (H1), and for each $\widehat{\mathbf{V}}_{i} \in \widehat{\mathcal{V}}$ we have

$$
\widehat{B}_{i}^{(j)}(s, t) \geq 0, \quad(s, t) \in \Omega_{0}, j=1,2,3 .
$$

Proof. We consider the two cases (30) and (31) separately.

- Let $\widehat{\mathbf{V}}_{i}=\mathbf{V}_{k} \in \mathcal{V}$. Since a refinement produces nested triangulations, the molecule associated to $\widehat{\mathbf{V}}_{i}$, as a vertex of $\widehat{\mathcal{T}}$, is contained in the molecule associated to $\mathbf{V}_{k}$, as a vertex of $\mathcal{T}$. Therefore, from $(\mathrm{H} 1)$ and (30), the PS triangle associated to $\widehat{\mathbf{V}}_{i}$, say $\widehat{T}_{i}$, contains the half molecule associated to $\widehat{\mathbf{V}}_{i}$. Hence, all the PS points of $\widehat{\mathbf{V}}_{i}$ belong to $\widehat{T}_{i}$, independently of the PS refinement of $\widehat{\mathcal{T}}$, and (32) follows from Theorem 1.

- If $\widehat{\mathbf{V}}_{i} \notin \mathcal{V}$, the molecule associated to $\widehat{\mathbf{V}}_{i}$ is contained in the triangle $\mathbf{V}_{i_{1}} \mathbf{V}_{i_{2}} \mathbf{V}_{i_{3}}$, see Fig. 8. Thus, from (31) it follows that the half molecule associated to $\widehat{\mathbf{V}}_{i}$ is contained in the corresponding PS triangle. Therefore, from Theorem 1 we obtain that $\widehat{B}_{i}^{(j)} \geq 0$.

In both cases $\widehat{\mathcal{T}}$ satisfies (H1).

Now we can state the main result of this section.

Proposition 7. Let $\mathbf{G}$ be a quadratic NURBS map as in (13). Let $\mathcal{T}$ be an admissible triangulation of $\Omega_{0}$ with a given $P S$ refinement. Then for any $P S$ refinement of any refinement $\widehat{\mathcal{T}}$ of $\mathcal{T}$ we can construct a set of nonnegative $P S$ B-splines

$$
\left\{\widehat{B}_{i}^{(j)}, i=1, \ldots, \widehat{N}_{V}, j=1,2,3\right\},
$$

so that

$$
\mathbf{G}(s, t)=\sum_{i=1}^{\widehat{N}_{V}} \sum_{j=1}^{3} \widehat{\mathbf{c}}_{i}^{(j)} \widehat{P}_{i}^{(j)}(s, t), \quad(s, t) \in \partial \Omega_{0},
$$


where

$$
\widehat{P}_{i}^{(j)}(s, t):=\frac{\widehat{\omega}_{i}^{(j)} \widehat{B}_{i}^{(j)}(s, t)}{\sum_{l=1}^{\widehat{N}_{V}} \sum_{r=1}^{3} \widehat{\omega}_{l}^{(r)} \widehat{B}_{l}^{(r)}(s, t)}, \quad \widehat{\omega}_{i}^{(j)}>0 .
$$

Proof. Let us consider a set of PS B-splines as in Proposition 3,

$$
\left\{B_{i}^{(j)}, i=1, \ldots, N_{V}, j=1,2,3\right\}
$$

for the triangulation $\mathcal{T}$ such that the boundary PS B-splines in (35) are determined according to (17) and (18).

Now let the boundary PS B-splines in (33) be determined according to (26) and (30)-(31) starting from the PS B-splines (35). From Proposition 6 it follows that the boundary PS B-splines in (33) are nonnegative. From Proposition 4 we know that the geometry map $\mathbf{G}$ can be exactly described on $\partial \Omega_{0}$ by a NURPS representation on $\widehat{\mathcal{T}}$, i.e. in the form (34). Therefore, we only need to prove that the weights $\widehat{\omega}_{i}^{(j)}$ in (34) are positive. To this end, we note that, after the refinement, the boundary PS B-splines in (33) can be associated either to old or new boundary vertices.

- According to (30), for old boundary vertices the PS triangles remain unchanged. Hence, by Proposition 5, the weights associated to the corresponding PS B-splines also remain the same, and so they are positive.

- For any new boundary vertex, $\widehat{\mathbf{V}}_{i}$, we can consider the subset of vertices in $\widehat{\mathcal{V}}$ consisting of the old vertices and $\widehat{\mathbf{V}}_{i}$ only, i.e. $\mathcal{V} \cup \widehat{\mathbf{V}}_{i}$, see Fig. 8. By (31), the PS triangle associated to $\widehat{\mathbf{V}}_{i}$ is determined using the same rule as in (18) with respect to this reduced sequence of boundary vertices. As a consequence, by Proposition 2, the weights $\widehat{\omega}_{i}^{(j)}, j=1,2$, agree with the corresponding weights of the NURBS map $\mathbf{G}$ represented on a new sequence of knots obtained by one step of the knot insertion process, see Proposition 4. So they are positive. Therefore, we obtain nonnegative B-splines and positive weights for a special subset of $\widehat{\mathcal{V}}$, namely $\mathcal{V} \cup \widehat{\mathbf{V}}_{i}$. If we keep the same PS triangle related to $\widehat{\mathbf{V}}_{i}$ when dealing with the whole set $\widehat{\mathcal{V}}$, then it follows from Proposition 5 that the same (positive) weights are retained in the NURPS representation (34).

The weight $\widehat{\omega}_{i}^{(3)}$ has no influence on the boundary. 

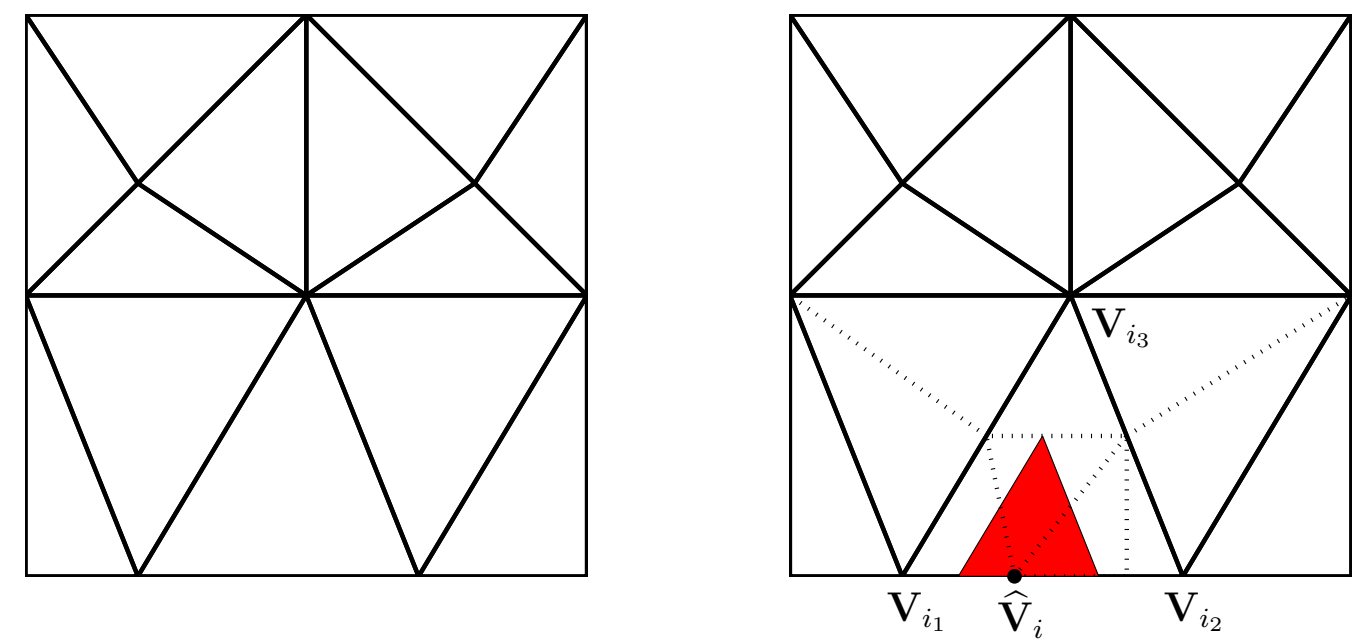

Figure 8: Left: a triangulation of the unit square. Right: a refined triangulation where the new edges are dashed. The PS triangle chosen as in (31) for the vertex $\widehat{\mathbf{V}}_{i}$ is shaded.

Summarizing, when we start with an admissible triangulation $\mathcal{T}$ of $\Omega_{0}$, it is possible to provide a NURPS representation of $\partial \Omega$ using positive weights and nonnegative PS B-splines. Moreover, an exact NURPS representation can be kept along any refinement of $\mathcal{T}$, still retaining positive weights and nonnegative PS B-splines. Referring to Remark 1, an appropriate initial triangulation could be a four directional triangulation where the horizontal and vertical edges coincide with the tensor-product knot lines of the given NURBS.

Finally, we remark that it is not necessary to stick to the PS triangles suggested in the proof of Proposition 7 along the boundary. This choice was simply considered to be able to provide a more readable proof. From a stability point of view, it is preferable to choose smaller PS triangles when possible. Let $\widehat{T}_{i}$ be the PS triangle of boundary vertex $\widehat{\mathbf{V}}_{i}$ constructed in the proof, then any smaller triangle $\widetilde{T}_{i} \subset \widehat{T}_{i}$ containing the PS points of $\widehat{\mathbf{V}}_{i}$ will also be a valid choice, resulting in a NURPS representation of $\partial \Omega$ with positive weights and nonnegative PS B-splines. This can be easily shown by referring to the tangent property of the control triangle defined by (22) to the weight function $D_{N}$ in (20), see also Fig. 9.

Note that these smaller PS triangles do not necessarily satisfy (H1) anymore. So, in case another refinement step is required one should go back to the 


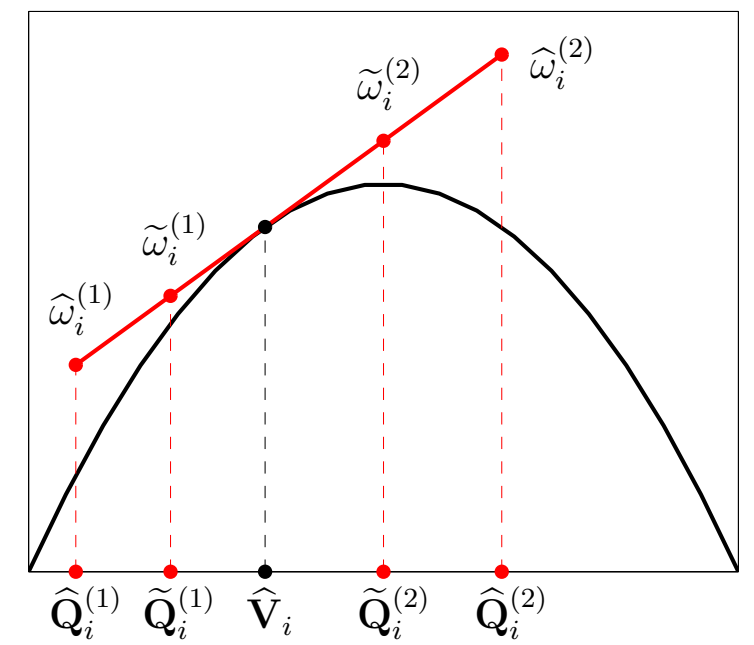

Figure 9: Vertical cross section of two PS control triangles along the boundary, defined by $\left(\widehat{\mathbf{Q}}_{i}^{(j)}, \widehat{\omega}_{i}^{(j)}\right)$ and $\left(\widetilde{\mathbf{Q}}_{i}^{(j)}, \widetilde{\omega}_{i}^{(j)}\right), j=1,2,3$, which are both tangent to the weight function $D_{N}$ at boundary vertex $\widehat{\mathbf{V}}_{i}$.

original PS triangle (and possibly shrink it again according to the new refined triangulation).

\section{Numerical examples}

In this section we illustrate how the presented conversion strategy performs by means of three examples. In all cases the PS refinements of the considered triangulations are based on barycenters.

\subsection{Example 1: NURPS description of a planar NURBS domain}

This example shows the effectiveness of the proposed strategy in converting a NURBS domain into a NURPS form. Let us consider the domain $\Omega$ given as image of the unit square by a quadratic NURBS map as in (13) defined by the knots

$$
\begin{aligned}
& \mathbf{u}:=\left\{u_{1}=u_{2}=u_{3}=0, u_{4}=u_{5}=u_{6}=1\right\}, \\
& \overline{\mathbf{u}}:=\left\{\bar{u}_{1}=\bar{u}_{2}=\bar{u}_{3}=0, \bar{u}_{4}=0.5, \bar{u}_{5}=\bar{u}_{6}=\bar{u}_{7}=1\right\},
\end{aligned}
$$

and by the control points and weights given in Table 1, see also Fig. 10 (left). The boundary of the obtained domain, see Fig. 10 (right), consists of three straight line segments and of segments of an ellipse and a hyperbola. 

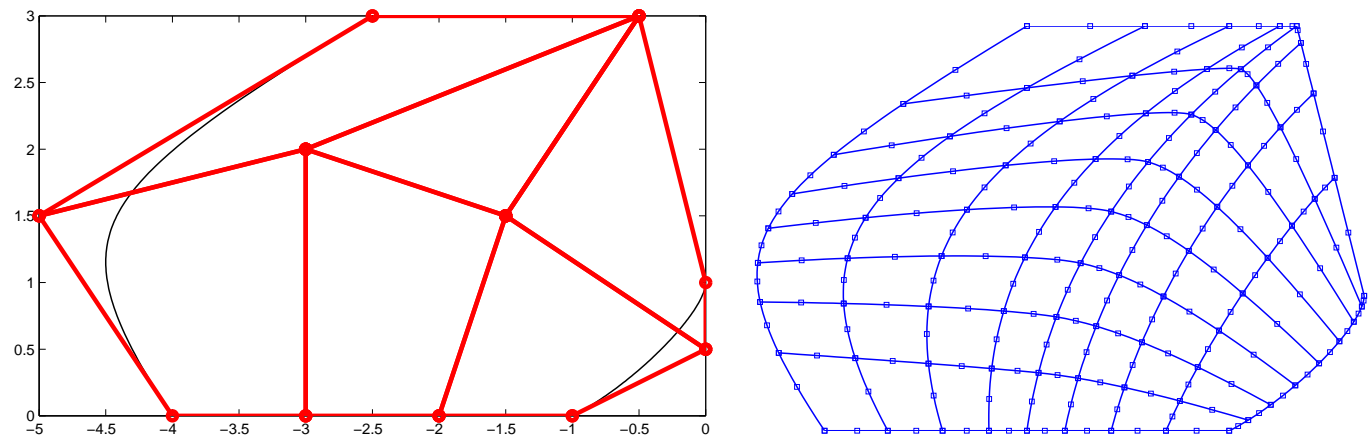

Figure 10: Example 1. Left: the control net and the boundary of the described domain $\Omega$. Right: isoparametric lines of the NURBS map.

\begin{tabular}{c|cc|cc|cc|cc}
$i$ & $\mathbf{p}_{i, 1}$ & $w_{i, 1}$ & $\mathbf{p}_{i, 2}$ & $w_{i, 2}$ & $\mathbf{p}_{i, 3}$ & $w_{i, 3}$ & $\mathbf{p}_{i, 4}$ & $w_{i, 4}$ \\
\hline 1 & $(-1,0)$ & 1 & $(-2,0)$ & 1 & $(-3,0)$ & 1 & $(-4,0)$ & 1 \\
2 & $(0,0.5)$ & $1 / 2$ & $(-1.5,1.5)$ & 1 & $(-3,2)$ & 1 & $(-5,1.5)$ & 2 \\
3 & $(0,1)$ & 1 & $(-0.5,3)$ & 1 & $(-0.5,3)$ & 1 & $(-2.5,3)$ & 1
\end{tabular}

Table 1: Example 1. Control points and weights of the quadratic NURBS geometry map.

The left column of Fig. 12 depicts some isoparametric lines of the NURPS map constructed according to the strategy described in Section 4, and defined on the triangulation of the parametric domain shown in Fig. 11 (left). Despite the very coarse triangulation, these lines are in quite good agreement with the corresponding isoparametric lines of the given NURBS map. By refining the triangulation, this agreement improves. The right column of Fig. 12 depicts the resulting NURPS image defined on the mildly refined triangulation shown in Fig. 11 (right).

\subsection{Example 2: NURPS approximation of a NURBS domain}

Since the procedure described in Section 4 works componentwise, it can be extended straightforwardly in case the object of interest is a two-dimensional manifold embedded in a multi-dimensional space. In particular, the case of curved surfaces in the three-dimensional setting is of salient interest. Of course, a general quadratic NURBS surface cannot be reproduced by a NURPS one, but the considered approach provides a very efficient and accurate NURPS approximation of the given NURBS geometry, still preserving the parametrization along the boundary. This is illustrated in the next example. The considered domain $\Omega$ is an approximation of a hyperboloid, and it has been inspired by the example in Subsection 3.2 in [2]. It is given as 

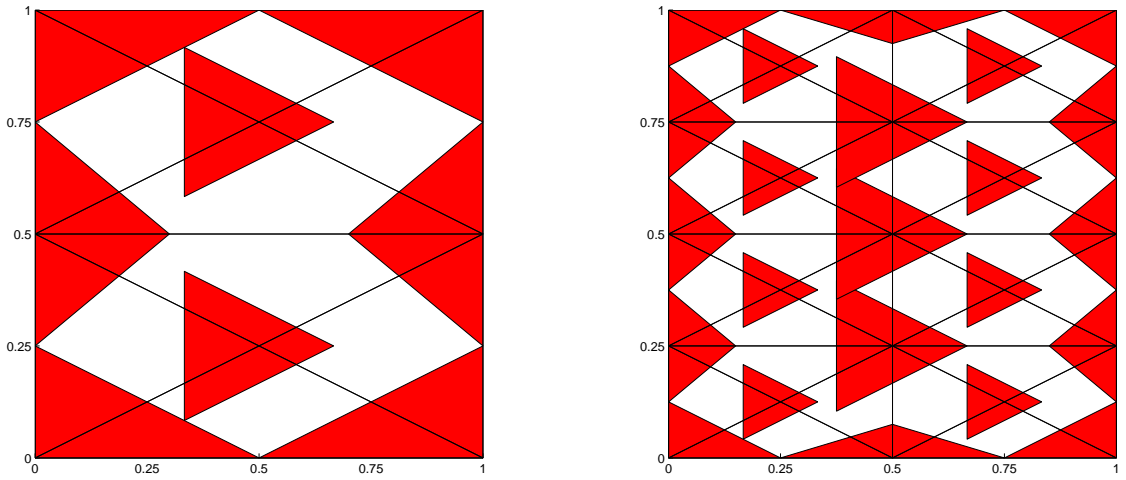

Figure 11: Example 1. Two triangulations of $\Omega_{0}$ and corresponding PS triangles (shaded).
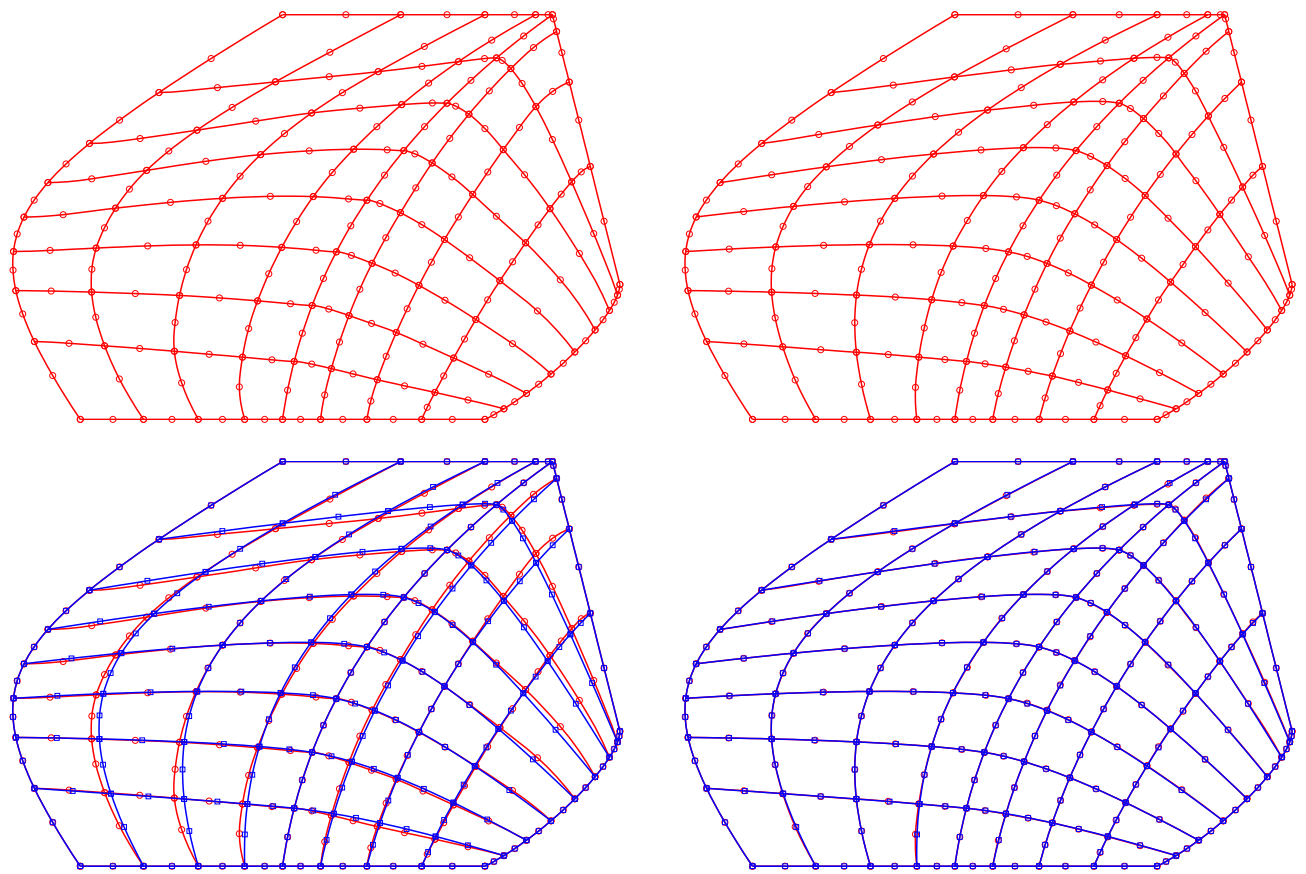

Figure 12: Example 1. Top: isoparametric lines of the NURPS maps corresponding to the triangulations shown in Fig. 11. Bottom: superimposed isoparametric lines of the NURBS map (blue squares) and of the NURPS maps (red circles) corresponding to the triangulations in Fig. 11. 

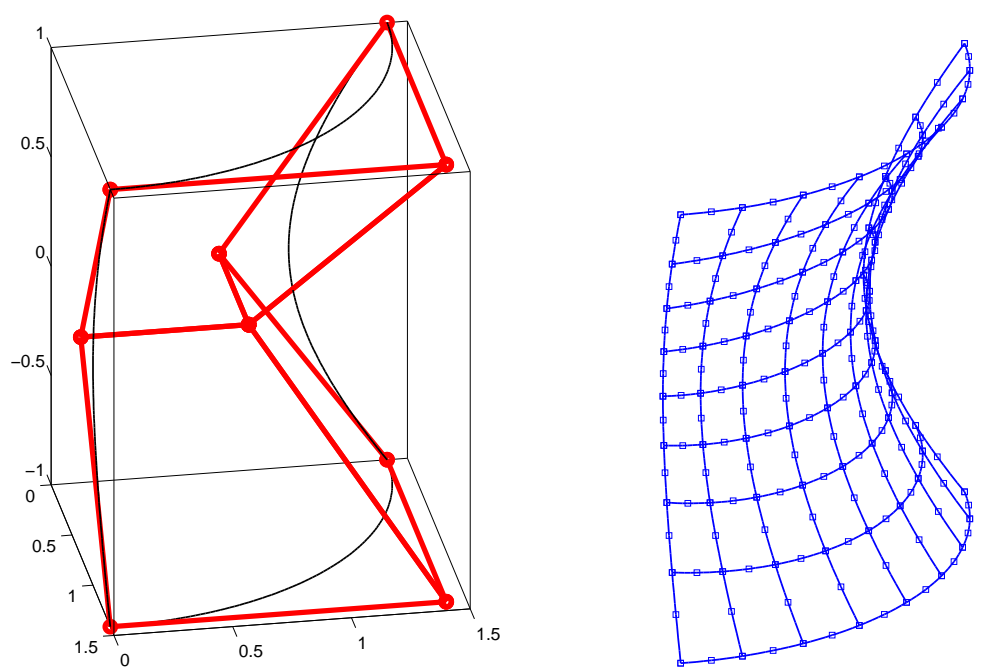

Figure 13: Example 2. Left: the control net and the boundary of the described domain $\Omega$. Right: isoparametric lines of the NURBS map.

\begin{tabular}{c|cc|cc|cc}
$i$ & $\mathbf{p}_{i, 1}$ & $w_{i, 1}$ & $\mathbf{p}_{i, 2}$ & $w_{i, 2}$ & $\mathbf{p}_{i, 3}$ & $w_{i, 3}$ \\
\hline 1 & $(\sqrt{2}, 0,1)$ & 1 & $(\sqrt{2}, \sqrt{2}, 1)$ & $\sqrt{2} / 2$ & $(0, \sqrt{2}, 1)$ & 1 \\
2 & $(\sqrt{2} / 2,0,0)$ & $\sqrt{2}$ & $(\sqrt{2} / 2, \sqrt{2} / 2,0)$ & $1 / 2$ & $(0, \sqrt{2} / 2,0)$ & $\sqrt{2}$ \\
3 & $(\sqrt{2}, 0,-1)$ & 1 & $(\sqrt{2}, \sqrt{2},-1)$ & $\sqrt{2} / 2$ & $(0, \sqrt{2},-1)$ & 1
\end{tabular}

Table 2: Example 2. Control points and weights of the quadratic NURBS geometry map.

image of the unit square by a quadratic NURBS map as in (13) defined by the knots

$$
\begin{aligned}
& \mathbf{u}:=\left\{u_{1}=u_{2}=u_{3}=0, u_{4}=u_{5}=u_{6}=1\right\}, \\
& \overline{\mathbf{u}}:=\left\{\bar{u}_{1}=\bar{u}_{2}=\bar{u}_{3}=0, \bar{u}_{4}=\bar{u}_{5}=\bar{u}_{6}=1\right\},
\end{aligned}
$$

and by the control points and weights given in Table 2, see also Fig. 13 (left). The NURBS patch is bounded by two arcs of circles and two arcs of hyperbolas.

The left column of Fig. 15 depicts two different views of isoparametric lines of the given NURBS map superimposed to those of the NURPS map defined on the coarse triangulation of the parametric domain shown in Fig. 14 (left). As for the planar domain considered in the previous example, these lines are in quite good agreement with the corresponding isoparametric lines of the given NURBS map. The right column of Fig. 15 depicts the resulting NURPS image defined on the mildly refined triangulation shown in Fig. 14 (right). The agreement of the isoparametric lines greatly improved. 

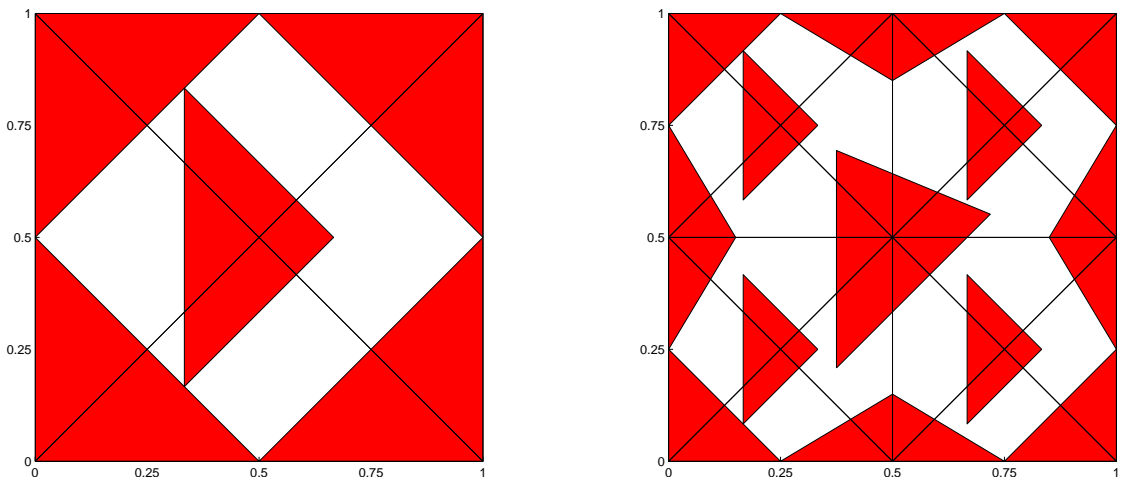

Figure 14: Example 2. Two triangulations of $\Omega_{0}$ and corresponding PS triangles (shaded).
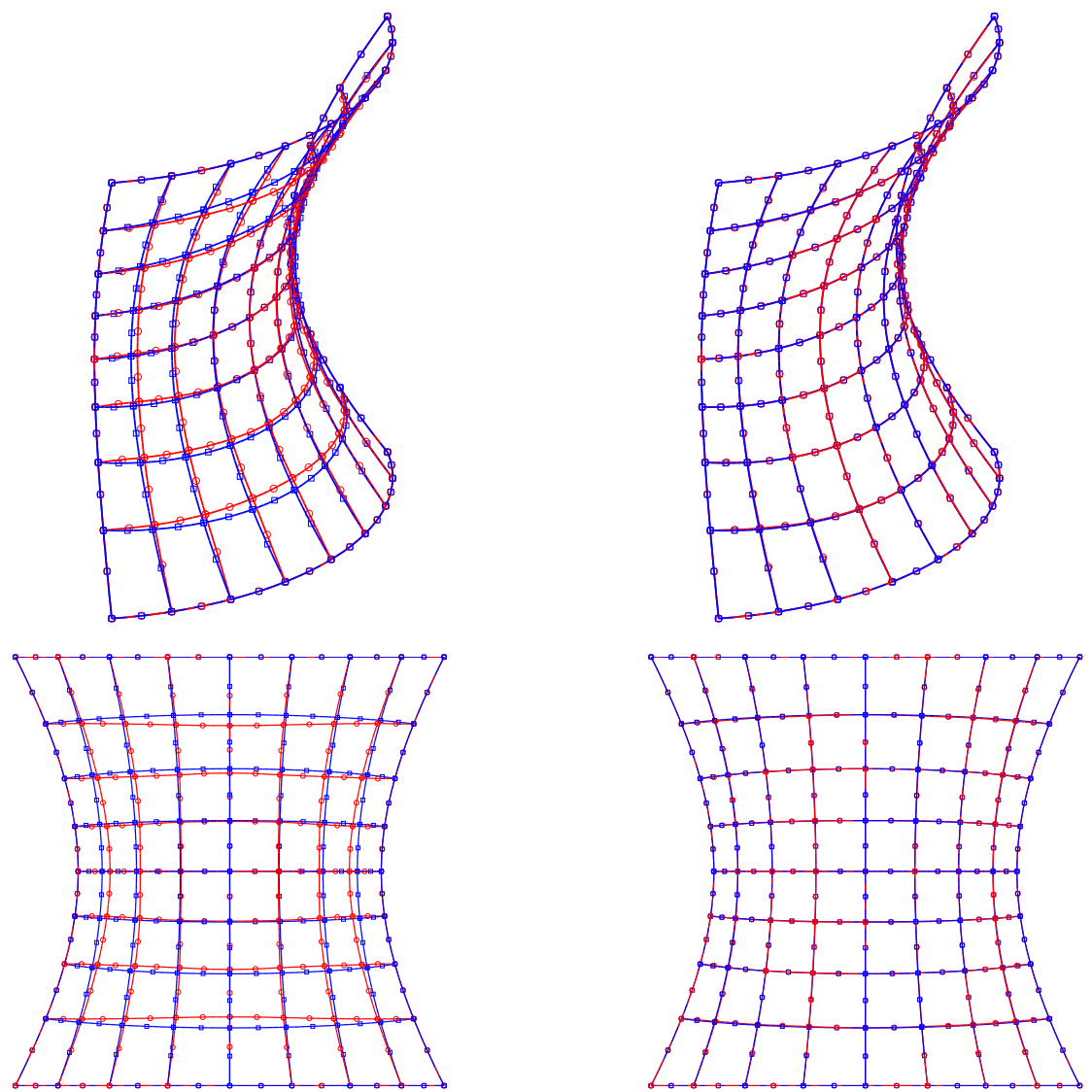

Figure 15: Example 2. Different views of superimposed isoparametric lines of the NURBS map (blue squares) and of the NURPS maps (red circles) corresponding to the triangulations in Fig. 14. 


\subsection{Example 3: analysis of a problem in solid mechanics based on NURPS splines}

This example aims to illustrate our conversion strategy in the presence of local refinements, by solving a classical problem in solid mechanics $[4,9,13]$. We consider an infinite plate with a circular hole of radius $R$, subject to an inplane uniform tension $T_{x}$ in $x$-direction, see Fig. 16 (left). For a homogeneous and isotropic material this problem features an exact solution which can be found in [8], Subsection 7.6. The infinite plate is modeled by a finite circular domain with radius $L$. Due to the symmetry, the computational domain $\Omega$ is restricted to a quarter, see Fig. 16 (right). We study the linear elastic behavior of the displacement field $\mathbf{v}: \Omega \rightarrow \mathbb{R}^{2}$ described by

$$
\operatorname{div} \sigma(\mathbf{v})=0 \text { in } \Omega .
$$

The exact solution is applied as a Neumann boundary condition, see Fig. 16 (right). For the sake of completeness we recall that $\sigma(\mathbf{v}):=\left\{\sigma_{i j}(\mathbf{v})\right\}_{i, j=1,2}$ with

$$
\begin{aligned}
\sigma_{i j}(\mathbf{v}):=\lambda \operatorname{div}(\mathbf{v}) \delta_{i j}+2 \mu \epsilon_{i j}(\mathbf{v}), \quad \epsilon_{i j}(\mathbf{v}):=\frac{1}{2}\left(\frac{\partial v_{i}}{\partial x_{j}}+\frac{\partial v_{j}}{\partial x_{i}}\right), \quad i, j=1,2, \\
\mathbf{v}:=\left(v_{1}, v_{2}\right), \quad(x, y):=\left(x_{1}, x_{2}\right) \\
\lambda:=\frac{E \nu}{(1+\nu)(1-2 \nu)}, \quad \mu:=\frac{E}{2(1+\nu)},
\end{aligned}
$$

where $E$ denotes the Young's modulus and $\nu$ the Poisson's ratio $[8,18]$.

In our computed example we have taken

$$
R=1, L=4, E=10^{5}, \nu=0.3, T_{x}=10 .
$$

Without the hole, the stress would be uniform

$$
\sigma_{1,1}=T_{x}, \quad \sigma_{1,2}=\sigma_{2,2}=0
$$

This distribution will alter only in the vicinity on the hole. More precisely, we get a peak stress concentration at the upper side of the hole: the stress component $\sigma_{1,1}$ takes the value 30 at the point $(0,1)$, see [8] and Fig. 17 (right).

We approximate the solution by an isogeometric analysis approach: first we describe the physical domain by a geometry map, and then we consider 

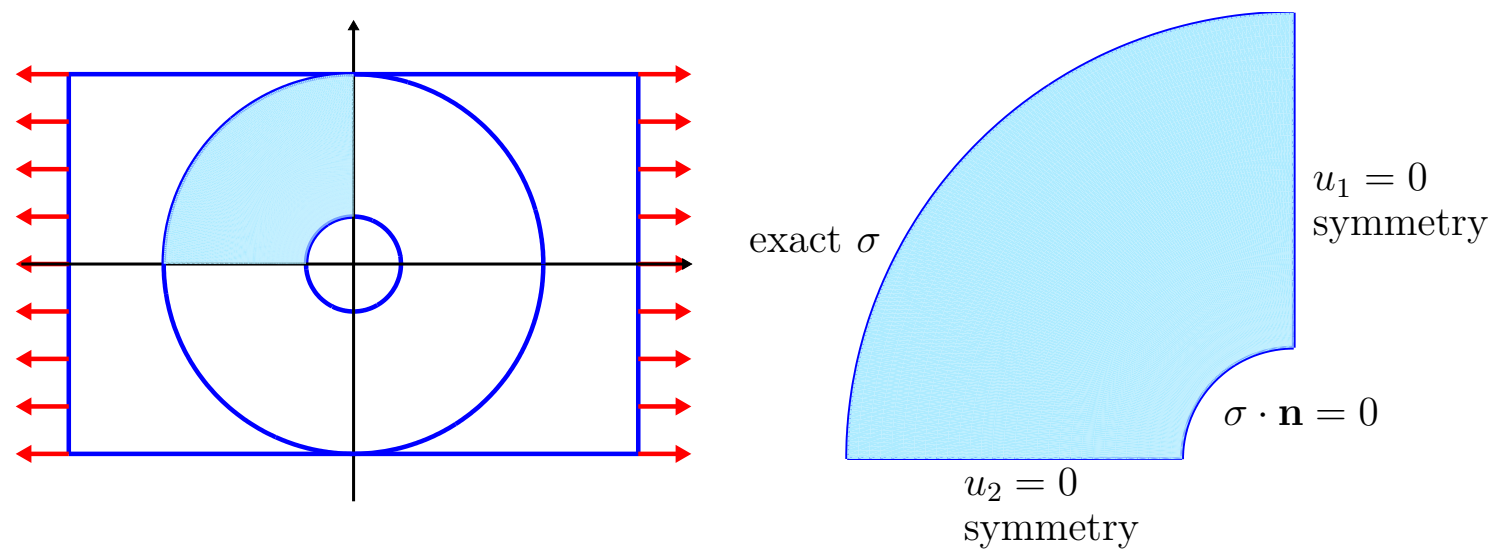

Figure 16: Example 3. Elastic plate with a circular hole: problem setting.

\begin{tabular}{c|cc|cc|cc}
$i$ & $\mathbf{p}_{i, 1}$ & $w_{i, 1}$ & $\mathbf{p}_{i, 2}$ & $w_{i, 2}$ & $\mathbf{p}_{i, 3}$ & $w_{i, 3}$ \\
\hline 1 & $(-1,0)$ & 1 & $(-2.5,0)$ & 1 & $(-4,0)$ & 1 \\
2 & $(-1,1)$ & $\sqrt{2} / 2$ & $(-2.5,2.5)$ & 1 & $(-4,4)$ & $\sqrt{2} / 2$ \\
3 & $(0,1)$ & 1 & $(0,2.5)$ & 1 & $(0,4)$ & 1
\end{tabular}

Table 3: Example 3. Control points and weights of the NURBS geometry map.

a Galerkin discretization of the weak form of the problem, based on the same functions used in the description of the geometry, see [9, 13] for a short summary.

The physical domain $\Omega$ is a quarter of an annulus, so it can be exactly described by a quadratic NURBS map as in (13) defined by the knots given in (36) and by the control points and weights given in Table 3, see also Fig. 17 (left).

Starting from the triangulation of the parametric domain $\Omega_{0}$ as depicted in Fig. 18 (left), we approximate the NURBS map by a NURPS map as described in Section 4, and then we compute an approximate solution by a Galerkin discretization based on the corresponding NURPS splines.

To improve the accuracy of the solution, successive local refinements of the triangulations are performed by applying an algorithm developed by Rivara, see $[19,23]$. The algorithm is based on the bisection of a local set of triangles by the longest side. It terminates in a finite number of steps and guarantees that no numerically degenerate triangles can be created. Since the exact solution is known, our adaptive refinements are simply driven by evaluating the error at the vertices of the PS refinement of the considered triangulations. 

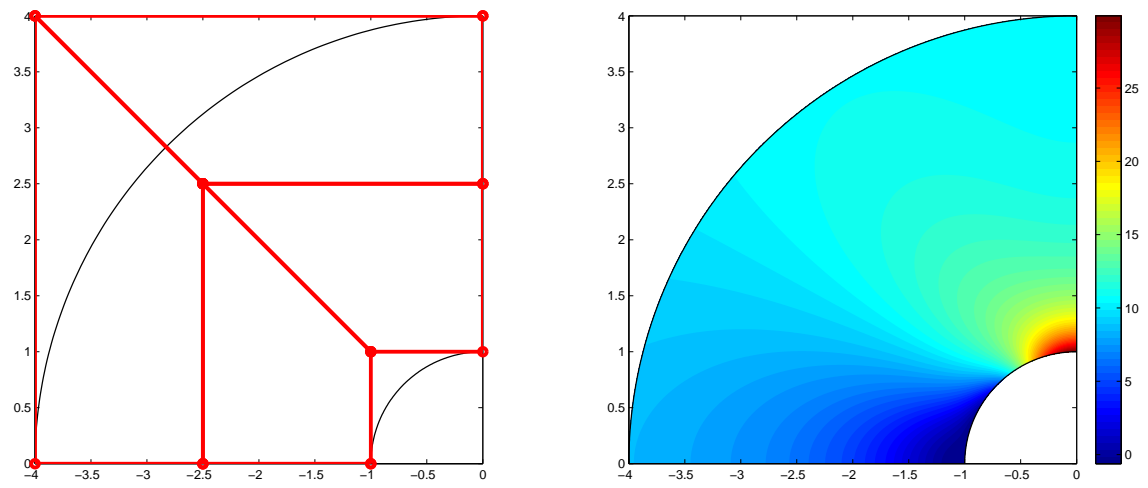

Figure 17: Example 3. Left: NURBS control net and physical domain. Right: exact $\sigma_{1,1}$.
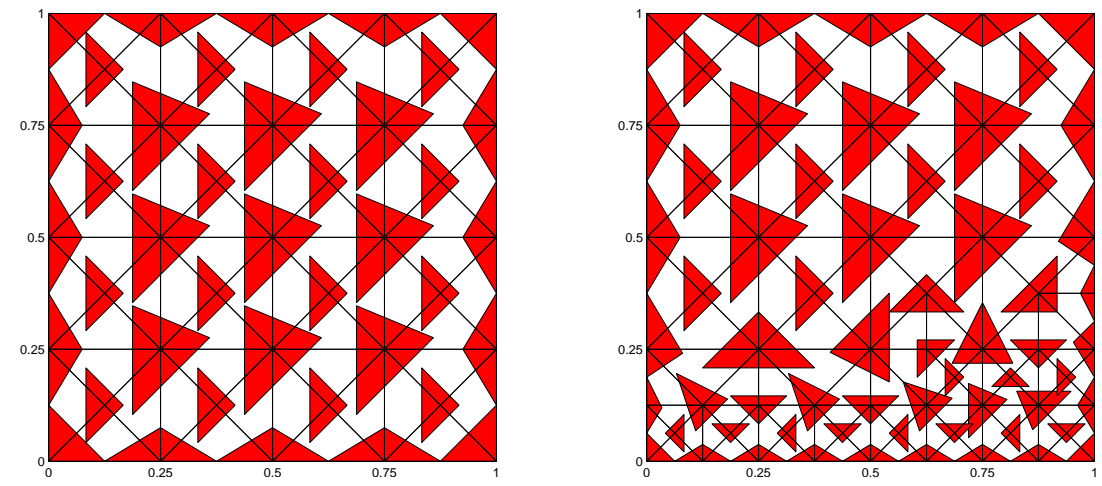

Figure 18: Example 3. Left: initial triangulation of the parametric domain with PS triangles. Right: a refined triangulation of the parametric domain with PS triangles.

The exact geometry is preserved at any level of the refinement, as well as nonnegativity of PS B-splines and positivity of weights in the NURPS forms. Since the initial triangulation is uniform, the refinement algorithm we used ensures that only isosceles right-angled triangles are produced along all the refinements, see for example Fig. 19 (left). As a consequence, the construction of boundary PS triangles as in (17)-(18), applied at each level, is sufficient to maintain nonnegativity of boundary PS B-splines along the entire process, see for example Fig. 18 (right). Therefore, this strategy has been used in the numerical tests. Figure 19 shows some NURPS approximations of the stress component $\sigma_{1,1}$ defined on locally refined triangulations.

Table 4 collects the approximated values for $\sigma_{1,1}$ at the upper side of the hole, together with the needed degrees of freedom and the correspond- 

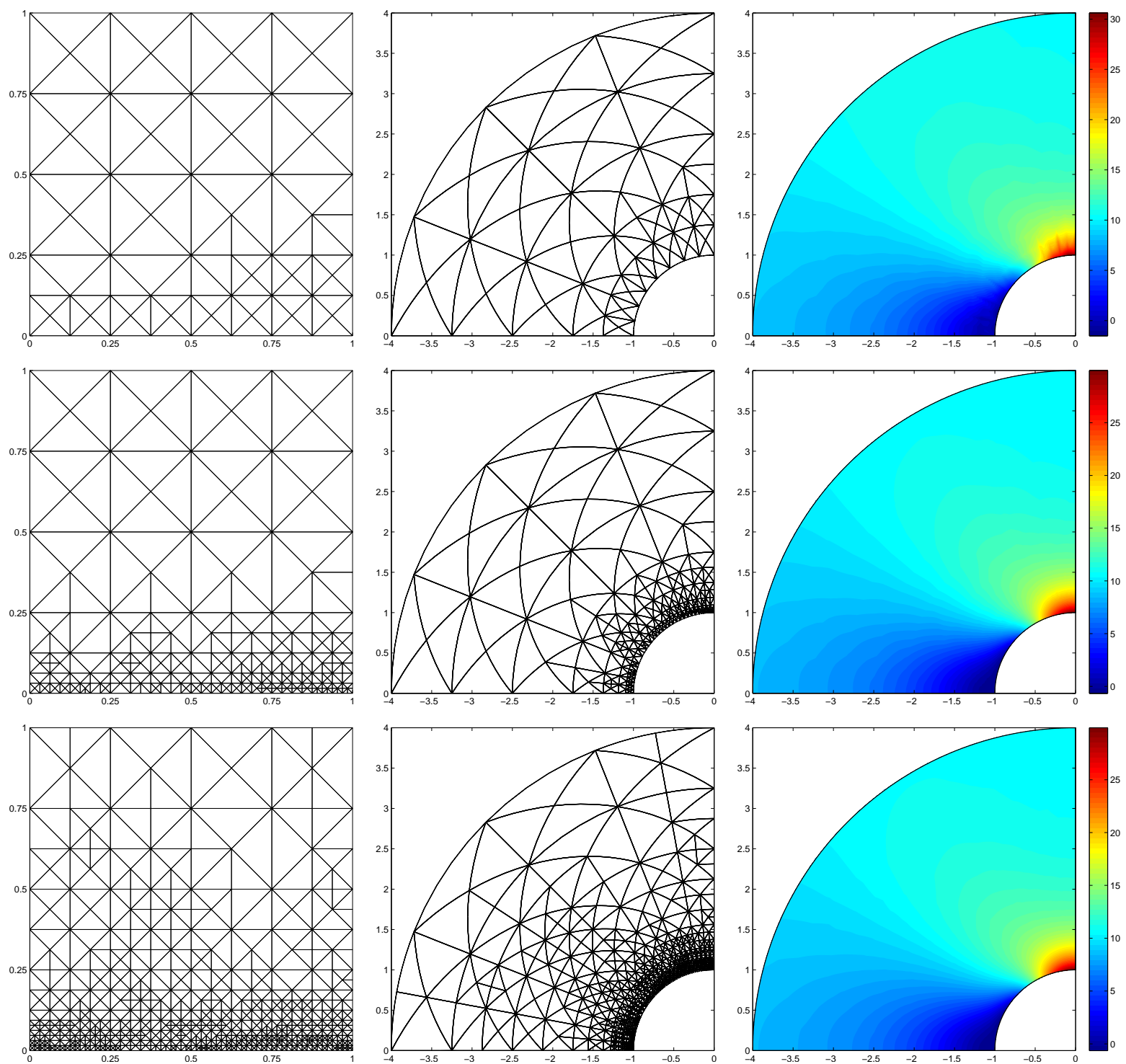

Figure 19: Example 3. Several locally refined triangulations of the parametric domain (left), the corresponding meshes in the physical domain (center), and the NURPS approximations of $\sigma_{1,1}$ (right). The top row corresponds to the triangulation and the PS triangles given in Fig. 18 (right). 


\begin{tabular}{r|r|r|r|r|r}
\multicolumn{3}{c|}{ Local refinements } & \multicolumn{3}{c}{ Global refinements } \\
\hline dof & max error & value at $(0,1)$ & dof & max error & value at $(0,1)$ \\
\hline 226 & 3.0872 & 28.6194 & 226 & 3.0872 & 28.6194 \\
$\mathbf{3 5 8}$ & $\mathbf{1 . 7 0 5 4}$ & $\mathbf{2 9 . 4 5 3 1}$ & 834 & 1.7044 & 29.4551 \\
580 & 0.6549 & 29.8116 & 3202 & 0.6548 & 29.8080 \\
$\mathbf{1 2 6 8}$ & $\mathbf{0 . 1 8 6 8}$ & $\mathbf{3 0 . 0 0 2 3}$ & 12546 & 0.2077 & 29.9412 \\
$\mathbf{3 7 2 2}$ & $\mathbf{0 . 0 5 2 9}$ & $\mathbf{2 9 . 9 9 7 1}$ & 49666 & 0.0587 & 29.9836
\end{tabular}

Table 4: Example 3. The number of degrees of freedom (dof), the max error on $\Omega$, and the approximate value at $(0,1)$ of $\sigma_{1,1}$ for different locally refined triangulations and uniform four directional triangulations. The boldfaced results correspond to the three triangulations shown in Fig. 19.

ing maximum error evaluated on a fine regular grid consisting of $400 \times 400$ points in the parameter domain $\Omega_{0}$. The results are given for locally refined triangulations (first three columns) as well as for uniform four directional triangulations (last three columns). The locally refined meshes in the first two rows are depicted in Fig. 18, and the results in boldface are illustrated in Fig. 19. The sequence of uniform four directional meshes are obtained by halving the mesh size in each step. As expected, local mesh refinement allows us to get the same accuracy with less degrees of freedom, see Table 4 and also the graph in Figure 20.

Further results for this problem in the context of $\operatorname{IgA}$ can be found in $[4,9,10,13,27]$. We refer to [25] for another example of NURPS based IgA addressing an advection dominated problem.

\section{Conclusions}

Quadratic Powell-Sabin splines and their rational extension, the so-called NURPS surfaces, are an interesting alternative for classical tensor-product NURBS, because they allow the use of local refinements while retaining a B-spline like representation and exact description of conic sections.

Tensor-product NURBS are the undisputed standard in commercial CAD systems, which motivated their use in the context of IgA. Recently, quadratic PS splines have been proposed as an alternative to classical tensor-product NURBS in IgA, mainly because of their flexibility with respect to local refinements: a major request in numerical treatment of partial differential equations. To completely frame the use of PS splines in IgA, in this paper we have presented a simple and effective strategy to convert a given planar geometry 


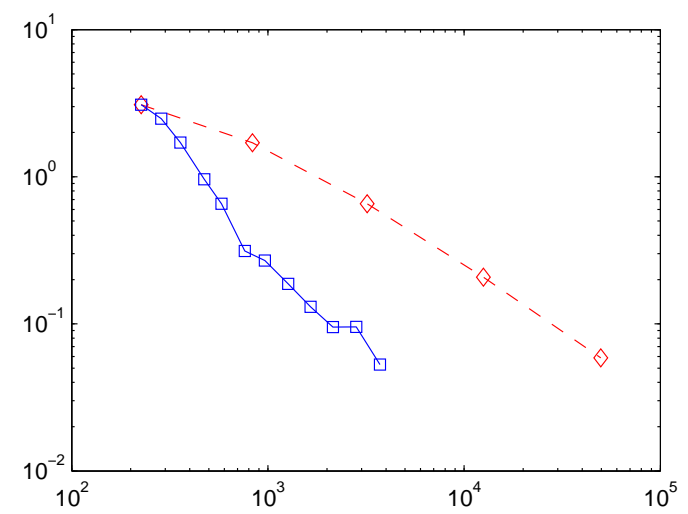

Figure 20: Example 3. Max errors of the approximations of the stress component $\sigma_{11}$ versus the number of degrees of freedom, using locally refined triangulations (solid blue) and uniform four directional triangulations (dashed red).

described by a quadratic NURBS representation into a NURPS representation, suitable for analysis.

The conversion process produces a new geometry map based on nonnegative PS B-splines and positive weights, and it provides a NURPS parametrization of the domain which is exact on the boundary. Moreover, due to the approximation properties of PS splines and to the smoothness of the quadratic NURBS representation, the obtained NURPS parametrization closely mimics the given NURBS parametrization in the interior, as soon as a reasonably fine triangulation of the parametric domain is considered. Therefore, if the provided NURBS parametrization is appropriate for the analysis, see, e.g., [31], the same holds for the NURPS one. Moreover, we proved that the initial planar geometry can be exactly preserved along possible (local) triangulation refinements by a NURPS representation based on nonnegative PS B-splines and positive weights.

The effectiveness of the method has been tested by considering a NURPS based IgA for a classical problem in solid mechanics, see also [25] for another example.

Our approach can be immediately extended to curved surfaces, see Subsection 6.2. Though a general quadratic NURBS surface cannot be exactly described by a NURPS form, the considered approach provides a very accurate NURPS approximation of a given non-planar NURBS geometry, still preserving the parametrization along the boundary. 
Due to their interest in applications we have focused on quadratic NURBS geometries. Of course, if higher order NURBS are of interest, quadratic PS splines cannot be used anymore.

Splines of higher degree and smoothness can also be defined on triangulations with a PS refinement, and they can be represented in a similar way as in the quadratic case, see [21] for $C^{2}$ quintics and [22] for a family of splines with arbitrary smoothness $r$ and degree $3 r-1$. Such spline spaces can be used to perform a similar conversion process, possibly by considering an appropriate degree elevation for the NURBS map.

The above splines present an additional smoothness $2 r-1$ on segments of the boundary consisting of collinear edges. If an even smoothness is required along the boundary, other spline spaces could be used. For example, we refer to [11] for the relevant $C^{2}$ case.

\section{References}

[1] Bazilevs Y., Calo V.M., Cottrell J.A., Evans J.A., Hughes T.J.R., Lipton S., Scott M.A., Sederberg T.W.; Isogeometric analysis using T-splines, Comput. Methods Appl. Mech. Engrg. 199 (2010), 229-263.

[2] Cottrell J.A., Hughes T.J.R., Reali A.; Studies of refinement and continuity in isogeometric structural analysis, Comput. Methods Appl. Mech. Engrg. 196 (2007), 4160-4183.

[3] de Boor C.; A Practical Guide to Spline, Springer, Revised ed., 2001.

[4] Dörfel M., Jüttler B., Simeon B.; Adaptive isogeometric analysis by local $h$-refinement with T-splines, Comput. Methods Appl. Mech. Engrg. 199 (2010), 264-275.

[5] Dierckx P.; On calculating normalized Powell-Sabin B-splines, Comput. Aided Geom. Design 15 (1997), 61-78.

[6] Dierckx P., Van Leemput S., Vermeire T.; Algorithms for surface fitting using Powell-Sabin splines, IMA J. Numer. Anal. 12 (1992), 271-299.

[7] Dokken, T., Lyche, T., Pettersen, K. F.; Locally Refinable Splines over Box-Partitions, Technical Report SINTEF A22403, (2012).

[8] Gould P.L.; Introduction to Linear Elasticity, Springer Verlag, Berlin, 1999. 
[9] Hughes T.J.R., Cottrell J.A., Bazilevs Y.; Isogeometric analysis: CAD, finite elements, NURBS, exact geometry and mesh refinement, Comput. Methods Appl. Mech. Engrg. 194 (2005), 4135-4195.

[10] Kleiss S.K., Jüttler B., Zulehner W.; Enhancing isogeometric analysis by a finite element-based local refinement strategy, Comput. Methods Appl. Mech. Engrg. 213-216 (2012), 168-182.

[11] Lai, M.-J.; On $C^{2}$ quintic spline functions over triangulations of PowellSabin's type, J. Comput. Appl. Math. 73 (1996), 135-155.

[12] Manni C., Pelosi F., Sampoli M.L.; Isogeometric analysis in advectiondiffusion problems: tension splines approximation, J. Comput. Appl. Math. 236 (2011), 511-528.

[13] Manni C., Pelosi F., Sampoli M.L.; Generalized B-splines as a tool in isogeometric analysis, Comput. Methods Appl. Mech. Engrg. 200 (2011), 867-881.

[14] Manni C., Sablonnière P.; Quadratic spline quasi-interpolants on PowellSabin partitions, Adv. Comput. Math. 26 (2007), 283-304.

[15] Peña J.M.; Shape-Preserving Representations in Computer Aided Geometric Design, Nova Science Publishers. Inc., 1999.

[16] Piegl L., Tiller W.; The NURBS Book, Springer, 1997.

[17] Powell M.J.D., Sabin M.A.; Piecewise quadratic approximations on triangles, ACM Trans. Math. Software 3 (1977), 316-325.

[18] Quarteroni A.; Numerical Models for Differential Problems, Springer, 2009.

[19] Rivara M.C.; Algorithms for refining triangular grids suitable for adaptive and multigrid techniques, Int. J. Numer. Methods Eng. 20 (1984), $745-756$.

[20] Sablonnière P.; Error bounds for Hermite interpolation by quadratic splines on an $\alpha$-triangulation, IMA J. Numer. Anal. 7 (1987), 495-508.

[21] Speleers H.; A normalized basis for quintic Powell-Sabin splines, Comput. Aided Geom. Design 27 (2010), 438-457. 
[22] Speleers H.; Construction of normalized B-splines for a family of smooth spline spaces over Powell-Sabin triangulations, Constr. Approx. (2012), in press.

[23] Speleers H., Dierckx P., Vandewalle S.; Numerical solution of partial differential equations with Powell-Sabin splines, J. Comput. Appl. Math. 189 (2006), 643-659.

[24] Speleers H., Dierckx P., Vandewalle S.; Weight control for modelling with NURPS surfaces, Comput. Aided Geom. Design 24 (2007), 179186.

[25] Speleers H., Manni C., Pelosi F., Sampoli M.L.; Isogeometric analysis with Powell-Sabin splines for advection-diffusion-reaction problems, Comput. Methods Appl. Mech. Engrg. 221-222 (2012), 132-148.

[26] Vanraes E., Dierckx P., Bultheel A.; On the choice of the PS-triangles, Technical Report 353, Dept. Computer Science, K.U.Leuven, 2003.

[27] Vuong A.-V., Giannelli C., Jüttler B., Simeon B.; A hierarchical approach to adaptive local refinement in isogeometric analysis, Comput. Methods Appl. Mech. Engrg. 200 (2011), 3554-3567.

[28] Willemans K., Dierckx P.; Surface fitting using convex Powell-Sabin splines, J. Comput. Appl. Math. 56 (1994), 263-282.

[29] Windmolders J., Dierckx P.; From PS-splines to NURPS. In: A. Cohen, C. Rabut, L.L. Schumaker (Eds.), Proceedings of Curve and Surface Fitting. Saint-Malo, 1999. Vanderbilt University Press, pp. 45-54, 2000.

[30] Windmolders J., Dierckx P.; NURPS for special effects and quadrics. In: T. Lyche, L.L. Schumaker (Eds.), Proceedings of Mathematical Methods for Curves and Surfaces. Oslo, 2000. Vanderbilt University Press, pp. 527-534, 2001.

[31] Xu G., Mourrain B., Duvigneau R., Galligo A.; Parameterization of computational domain in isogeometric analysis: methods and comparison, Comput. Methods Appl. Mech. Engrg. 200 (2011), 2021-2031. 